\title{
Combined Influence of Waist and Hip Circumference on Risk of Death in a Large Cohort of European and Australian Adults
}

\begin{abstract}
Adrian J. Cameron, PhD*; Helena Romaniuk, PhD*; Liliana Orellana, PhD; Jean Dallongeville, PhD; Annette J. Dobson, PhD; Wojciech Drygas, MD; Marco Ferrario, MD; Jean Ferrieres, MD; Simona Giampaoli, MD; Francesco Gianfagna, MD; Licia lacoviello, PhD; Pekka Jousilahti, PhD; Frank Kee, MD; Marie Moitry, MD; Teemu J. Niiranen, MD; Andrzej Pająk, PhD; Luigi Palmieri, PhD; Tarja Palosaari, MSc; Männistö Satu, PhD; Abdonas Tamosiunas, PhD; Barbara Thorand, PhD; Ulla Toft, PhD; Diego Vanuzzo, MD; Salomaa Veikko, MD; Giovanni Veronesi, PhD; Tom Wilsgaard, PhD; Kari Kuulasmaa, PhD; Stefan Söderberg, PhD
\end{abstract}

BACKGROUND: Waist circumference and hip circumference are both strongly associated with risk of death; however, their joint association has rarely been investigated.

METHODS AND RESULTS: The MONICA Risk, Genetics, Archiving, and Monograph (MORGAM) Project was conducted in 30 cohorts from 11 countries; 90487 men and women, aged 30 to 74 years, predominantly white, with no history of cardiovascular disease, were recruited in 1986 to 2010 and followed up for up to 24 years. Hazard ratios were estimated using sex-specific Cox models, stratified by cohort, with age as the time scale. Models included baseline categorical obesity measures, age, total and high-density lipoprotein cholesterol, systolic blood pressure, antihypertensive drugs, smoking, and diabetes mellitus. A total of 9105 all-cause deaths were recorded during a median follow-up of 10 years. Hazard ratios for all-cause death presented J- or U-shaped associations with most obesity measures. With waist and hip circumference included in the same model, for all hip sizes, having a smaller waist was strongly associated with lower risk of death, except for men with the smallest hips. In addition, among those with smaller waists, hip size was strongly negatively associated with risk of death, with $\approx 20 \%$ more people identified as being at increased risk compared with waist circumference alone.

CONCLUSIONS: A more complex relationship between hip circumference, waist circumference, and risk of death is revealed when both measures are considered simultaneously. This is particularly true for individuals with smaller waists, where having larger hips was protective. Considering both waist and hip circumference in the clinical setting could help to best identify those at increased risk of death.

Key Words: hip circumference $\mathbf{m}$ mortality $\mathbf{m}$ obesity $\mathbf{m}$ waist circumference

$T$ he prevalence of obesity is high or rapidly increasing in most countries, with serious health and economic consequences. ${ }^{1}$ Body mass index (BMI) is the most commonly used measure of obesity; however, it does not capture the differential effects of adipose tissue from different parts of the body ${ }^{2-8}$ or visceral and subcutaneous adipose tissue. ${ }^{9}$ Body shape differences mean that people with the same $\mathrm{BMI}$ can vary widely in their body fat distribution. ${ }^{10}$ $\mathrm{BMI}$ also does not distinguish between fat mass and fat-free mass, the latter having a strong inverse relationship with morbidity and mortality. ${ }^{8}$ The cost of the

Correspondence to: Adrian J. Cameron, PhD, Global Obesity Centre, Institute for Health Transformation, Deakin University, 221 Burwood Hwy, Burwood, VIC 3125, Australia. E-mail: adrian.cameron@deakin.edu.au

Supplementary Materials for this article are available at https://www.ahajournals.org/doi/suppl/10.1161/JAHA.119.015189

*Prof Cameron and Dr Romaniuk contributed equally to this work.

For Sources of Funding and Disclosures, see page 13.

() 2020 The Authors. Published on behalf of the American Heart Association, Inc., by Wiley. This is an open access article under the terms of the Creative Commons Attribution-NonCommercial License, which permits use, distribution and reproduction in any medium, provided the original work is properly cited and is not used for commercial purposes.

JAHA is available at: www.ahajournals.org/journal/jaha 


\section{CLINICAL PERSPECTIVE}

\section{What Is New?}

- To the best of our knowledge, this is the most comprehensive investigation of the risk of death associated with different combinations of waist circumference and hip circumference, using data from >90 000 individuals (from 11 countries) who were followed up for up to 24 years.

\section{What Are the Clinical Implications?}

- Among those with smaller waists (who would not normally have been identified as being at higher risk of death on the basis of their body size), having smaller hips was strongly associated with increased risk of death.

- Considering both waist and hip circumference simultaneously identifies almost 20\% more people as being at higher risk of death compared with using waist circumference alone, and is a simple and cost-effective way of identifying body shapes associated with increased risk of premature death.

\section{Nonstandard Abbreviations and Acronyms}

$\begin{array}{ll}\text { ABSI } & \text { a body shape index } \\ \text { BMI } & \text { body mass index } \\ \text { CVD } & \text { cardiovascular disease } \\ \text { HC } & \text { hip circumference } \\ \text { MORGAM } & \text { MONICA Risk, Genetics, Archiving, } \\ & \text { and Monograph Project } \\ \text { WC } & \text { waist circumference } \\ \text { WHR } & \text { waist/hip ratio }\end{array}$

imaging methods required to accurately assess fat distribution is prohibitive in many settings. From a public health perspective, it is therefore important to identify simple anthropometric measures that reflect adipose tissue distribution and are closely related to morbidity and premature death.

Waist circumference (WC) and hip circumference $(\mathrm{HC})$ are commonly used and easily understood measures of abdominal (upper-body) and gluteofemoral (lower-body) body size, respectively. WC is primarily a measure of visceral/ectopic and subcutaneous adipose tissue around the waist, whereas $\mathrm{HC}$ measures both adipose tissue and lower-body muscle mass. Numerous studies have shown that larger WC is strongly related to morbidity and premature death, while there is some evidence that larger $\mathrm{HC}$ is protective for these same outcomes. $2,6,8,9,11$ The effects of different fat depots in the upper and lower body are increasingly explained by variation in lipid storage and release ${ }^{11,12}$ and the secretion of adipose tissuerelated proteins. ${ }^{5,11}$ Given their opposite relationships with metabolic health, the ratio of WC to $\mathrm{HC}$ (WHR) was conceptualized as an overall measure of obesity, ${ }^{13}$ with the waist/height ratio also proposed as a way of capturing the distribution of body fat. ${ }^{14,15}$ WHR and waist/height ratio have a simple interpretation when the relationship between the 2 variables is linear, but lack interpretation otherwise. ${ }^{16}$ Furthermore, they can be identical for individuals of vastly different body shape. ${ }^{17}$ There is no conclusive evidence that WC alone or WHR is more strongly related to risk of premature death than BMI. ${ }^{18,19}$ Both, however, are predictors of death when added to a model also including BMI, meaning they are clearly identifying different components of obesity-related risk. ${ }^{18}$ "A body shape index" (ABSI) that incorporates WC, height, and BMI has also been proposed in an attempt to identify an optimal body size measure associated with mortality. ${ }^{10}$

A 2013 systematic review identified only 5 studies predicting premature death using statistical models that included separate measures of both WC and HC. ${ }^{7}$ In each case, the model including both measures was superior to a model including only one of them. These were single population studies with either a maximum follow-up of 12 years or $<1500$ deaths.

We aimed to conduct a novel assessment of the joint association of $\mathrm{HC}$ and WC with all-cause and cardiovascular disease (CVD) mortality outcomes, stratified by sex, using a large, multicountry cohort with long follow-up. We also report on the association between other more commonly used measures of body shape with mortality.

\section{METHODS}

\section{Data Sharing}

Details of data sharing arrangements and access to MONICA Risk, Genetics, Archiving, and Monograph (MORGAM) Project data are described in the following article: https://doi.org/10.1093/ije/dyh327. Please also see http://www.thl.fi/morgam.

\section{Study Population}

The MORGAM Project is an ongoing multinational, collaborative study of prospective cohorts set up to investigate CVD. ${ }^{20}$ Participating centers in each country recruited cohorts by taking random samples of geographically defined populations at different time periods, with standardized risk factors measured at baseline at enrollment and participants followed up for death. Details of MORGAM cohorts and data quality assessments are documented online,,$^{21,22}$ with data 
harmonized according to the Monitoring of Trends and Determinants in Cardiovascular Disease (MONICA) and MORGAM manuals. ${ }^{21,23,24}$

Our study is based on data from 30 MORGAM cohorts from 17 participating centers located in 10 European countries plus Australia, which included 111318 participants recruited between 1986 and 2010 who were followed up for up to 24 years. Participants were excluded if they were aged $<30$ years $(n=6647 ; 6.0 \%)$ or $\geq 75$ years $(n=1863 ; 1.7 \%)$ at baseline, had missing information on follow-up period $(n=473 ; 0.42 \%)$, had history of CVD $(n=4916 ; 4.4 \%)$ or history of CVD was unknown ( $n=589 ; 0.53 \%)$, with some participants excluded for multiple reasons. Of the 97189 (87.3\%) participants eligible for this study, 6702 (6.9\%) were excluded as they had incomplete baseline data for CVD risk factors. Our final analysis sample included 90487 participants (42792 women), who were predominantly white, with a median follow-up period of 10 years. Cohort characteristics are summarized by participating center and sex in Table 1. Each MORGAM participating center was responsible for ethical approval and patient consent, according to local rules at the time of study enrollment.

\section{End Points}

Two end points were defined, all-cause and CVD death, via linkage to national or regional death registries. The follow-up of a person continued until the earliest one of the following events occurred: death; end of fixed follow-up period of the cohort; withdrew from study; or lost to follow-up. Only $1.1 \%$ of the analysis sample withdrew or was lost to followup. CVD death was defined as death from coronary heart disease or stroke, in addition to unclassifiable deaths where there was insufficient evidence for coronary origin. The diagnostic classification was based on validation of the cause of each death or on the International Classification of Diseases (ICD) codes of the routine death registration. Slight variation was present in $I C D$ codes used because of local ICD coding practices (see description of MORGAM cohorts 22 for more details).

\section{Baseline Measurements}

Participants' measures were collected at enrollment. Anthropometric measurements included weight in kilograms, and height, WC, and HC in meters. ${ }^{21} \mathrm{BMl}$ was calculated as weight/height ${ }^{2}$. WHR and waist/ height ratio were calculated as $\mathrm{WC} / \mathrm{HC}$ and $\mathrm{WC} /$ height, respectively. ABSI was calculated as follows: $\mathrm{WC} /\left(\mathrm{BMI}^{2 / 3} \times\right.$ height $\left.^{1 / 2}\right)$. Blood pressure was measured as the mean of the first 2 measurements taken in a sitting position using the right arm and using a standard or random zero sphygmomanometer, or an automated device, after a 5-minute rest, except in the United Kingdom and 3 French cohorts, in which blood pressure was measured only once using an automated device. ${ }^{21}$ Total serum cholesterol and high-density lipoprotein cholesterol were analyzed in serum or plasma samples by local laboratories. ${ }^{21}$ Diabetes mellitus, use of antihypertensive drugs, and smoking of cigarettes, cigars/cigarillos, or pipes were self-reported. History of CVD was identified from documentation (ie, population-based coronary event or stroke registers, person's medical records, a hospital discharge register, or other health information system) or self-reported history of myocardial infarction or stroke, including angina pectoris when the data collected did not permit its separation from myocardial infarction.

\section{Statistical Analysis}

Anthropometric measures were categorized into 6 groups based on sex-specific sample means and SDs of all available data ( $<-1$ SD below the mean, -1 to $<-0.5$ SDs below the mean, $\geq-0.5-\leq 0.5 \mathrm{SDs}$ from the mean, $>0.5-1$ SD above the mean, >1-2 SDs above the mean, and $>2$ SDs above the mean). The ranges of values for the categories are shown in Table S1.

Associations between the categorical anthropometric measures and the risk of all-cause and cardiovascular death were estimated separately for men and women using Cox proportional-hazards models, stratified by cohort, with age as the time scale, after partial and further adjustment. These associations were estimated in models including each individual anthropometric measure or a model including both WC and HC. Partially adjusted models included age at baseline $(<50,50-<55,55-<60,60-<65,65-<70$, and $70-<75$ years). Further adjusted models included age and cardiovascular risk factors in the current version of the Framingham Risk Score, ${ }^{25}$ all measured at baseline: log of total cholesterol ( $\mathrm{mmol} / \mathrm{L})$, log of highdensity lipoprotein cholesterol ( $\mathrm{mmol} / \mathrm{L})$, systolic blood pressure $(\mathrm{mm} \mathrm{Hg}$ ), taking hypertension drugs (yes/no), current daily smoker (yes/no), and diabetes mellitus (yes/no). In the further adjusted model, interactions between baseline age and baseline measures, including anthropometric measures, were tested and retained if $P<0.001$. Main and interaction effects were tested using the Wald test. For models including both WC and $\mathrm{HC}$, we estimated the hazard ratios only for feasible combinations of these body measurements (observed in $>0.1 \%$ of the sample [Table S2]). The proportionalhazards assumption was checked using Schoenfeld residuals.

We performed complete case analyses, including all participants who had no missing data for all baseline 


\begin{tabular}{|c|c|c|c|c|c|c|c|c|c|c|c|c|c|c|c|c|c|c|c|c|}
\hline \multirow{2}{*}{ 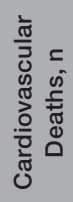 } & $\sum^{\bar{\Phi}}$ & $\approx$ & Бृ & 怘 & $\stackrel{N}{N}$ & $\underset{ \pm}{ \pm}$ & $\bar{m}$ & 号 & ๗్ల & $\infty$ & $\stackrel{d}{\sim}$ & 怘 & 怘 & 导 & $\mp$ & $\sim$ & б & 흐 & 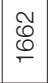 & \\
\hline & 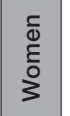 & $F$ & $\stackrel{\oplus}{\rightleftharpoons}$ & $\stackrel{\tilde{N}}{\stackrel{N}{*}}$ & & & & $\stackrel{\infty}{\stackrel{\infty}{\sigma}}$ & $\stackrel{\mathscr{T}}{\leftarrow}$ & $\infty$ & 으 & 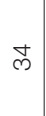 & $\neg$ & $\begin{array}{l}\stackrel{\circ}{\circ} \\
\stackrel{9}{2}\end{array}$ & 0 & - & $\infty$ & & $\frac{\circ}{6}$ & 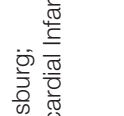 \\
\hline \multirow{2}{*}{ 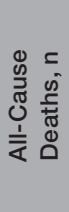 } & $\sum^{\frac{\sigma}{\omega}}$ & $\stackrel{\llcorner}{\infty}$ & $\stackrel{\substack{q \\
q}}{ }$ & 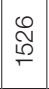 & 富 & \& & $\underset{\ddagger}{\mp}$ & 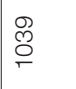 & $\begin{array}{l}\infty \\
\stackrel{0}{0}\end{array}$ & is & ฉ̀ & 兽 & $\left|\begin{array}{l}\infty \\
\stackrel{\leftrightarrow}{\leftarrow}\end{array}\right|$ & 足 & 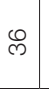 & $\bar{\sim}$ & $\stackrel{\substack{\sim \\
\sim}}{\sim}$ & ₹ & $\begin{array}{l}\infty \\
\infty \\
\infty \\
b\end{array}$ & \multirow{4}{*}{ 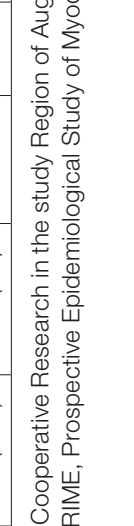 } \\
\hline & 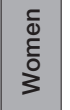 & f & \begin{tabular}{l}
\multirow{d}{0}{} \\
d
\end{tabular} & ల్ & & & & 웡 & के & $\bar{m}$ & $\stackrel{8}{\infty}$ & $\stackrel{\widehat{N}}{\sim}$ & $\stackrel{0}{\circ}$ & 8 & $\infty$ & $\simeq$ & $\stackrel{\Delta}{\sim}$ & & 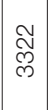 & \\
\hline \multirow{2}{*}{ 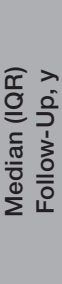 } & $\stackrel{\ulcorner}{\Sigma}$ & $\begin{array}{l}\widehat{a} \\
\dot{w} \\
\hat{\sim} \\
\hat{f}\end{array}$ & 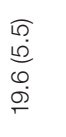 & $\begin{array}{l}\bar{o} \\
\dot{0} \\
\underline{0} \\
\dot{p} \\
\dot{p}\end{array}$ & 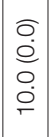 & 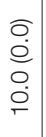 & $\begin{array}{l}\bar{\partial} \\
\dot{\dot{O}} \\
\dot{0} \\
\dot{\varphi}\end{array}$ & $\begin{array}{l}\widehat{\omega} \\
\infty \\
\omega \\
\omega \\
\stackrel{\infty}{\omega}\end{array}$ & 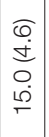 & \begin{tabular}{|c|c|}
$\hat{\sigma}$ \\
$\dot{\rho}$ \\
0 \\
$\dot{f}$ \\
$\dot{f}$
\end{tabular} & 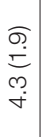 & 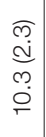 & 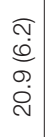 & 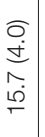 & 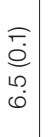 & 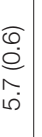 & 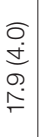 & 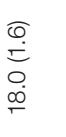 & $\begin{array}{l}\sigma \\
\dot{0} \\
\circ \\
\stackrel{\circ}{\circ}\end{array}$ & \\
\hline & 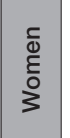 & $\begin{array}{l}\widetilde{\vec{\omega}} \\
\stackrel{\omega}{\omega 0} \\
\stackrel{+}{\dot{\alpha}}\end{array}$ & 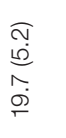 & 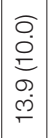 & & & & 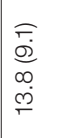 & 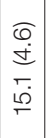 & \begin{tabular}{|c|} 
\\
$\dot{\rho}$ \\
$\dot{\rho}$ \\
0 \\
$\dot{f}$ \\
\end{tabular} & 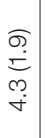 & 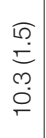 & 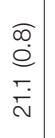 & 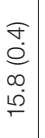 & 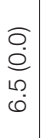 & 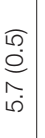 & 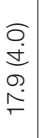 & & $\begin{array}{l}\sigma \\
0 \\
\dot{0} \\
0 \\
0 \\
\dot{\sigma}\end{array}$ & \\
\hline \multirow{2}{*}{ 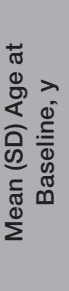 } & $\stackrel{c}{\stackrel{c}{\Phi}}$ & 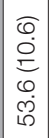 & 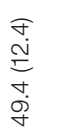 & 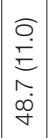 & $\begin{array}{l}\widehat{\sigma} \\
\stackrel{d}{\sim} \\
\hat{j} \\
\dot{\omega}\end{array}$ & $\begin{array}{l}\widehat{\alpha} \\
\dot{d} \\
\vec{d} \\
\dot{d} \\
\dot{b}\end{array}$ & 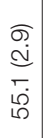 & 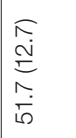 & 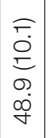 & 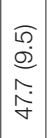 & 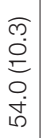 & 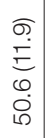 & 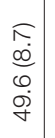 & 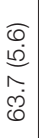 & $\begin{array}{c}\widehat{0} \\
\infty \\
\infty \\
\infty \\
\dot{q}\end{array}$ & 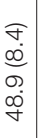 & 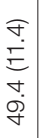 & 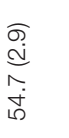 & 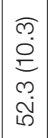 & $\begin{array}{l}5 \\
\frac{5}{5} \\
\frac{5}{5} \\
5 \\
5\end{array}$ \\
\hline & 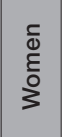 & 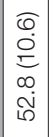 & 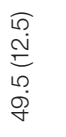 & 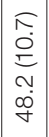 & & & & 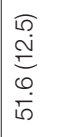 & 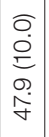 & 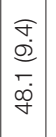 & 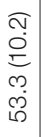 & 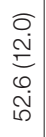 & $\mid \begin{array}{c}0 \\
0 \\
\infty \\
\infty \\
\sigma \\
\sigma \\
\end{array}$ & 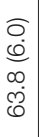 & $\begin{array}{l}\overrightarrow{0} \\
\stackrel{\infty}{\hat{\alpha}} \\
\hat{\sigma}\end{array}$ & 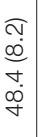 & 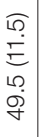 & & 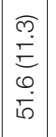 & . \\
\hline \multirow{2}{*}{ 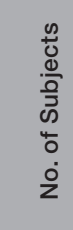 } & $\sum_{\Sigma}^{\frac{\sigma}{\omega}}$ & $\begin{array}{l}\infty \\
o \\
\sigma \\
\end{array}$ & 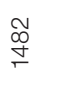 & 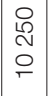 & $\begin{array}{l}\stackrel{\infty}{N} \\
\tilde{N}\end{array}$ & $\begin{array}{l}\text { Oे } \\
\text { d }\end{array}$ & $\underset{\mathbb{N}}{\underset{\mathbb{N}}{\sim}}$ & $\begin{array}{l}8 \\
0 \\
0 \\
i\end{array}$ & $\begin{array}{l}\stackrel{0}{\sim} \\
\stackrel{\sim}{v}\end{array}$ & $\begin{array}{l}\frac{L}{2} \\
\frac{1}{6}\end{array}$ & $\begin{array}{l}\text { 足 } \\
\text { 员 }\end{array}$ & $\underset{\mathcal{N}}{\leftarrow}$ & $\mid \begin{array}{l}0 \\
0 \\
1\end{array}$ & $\begin{array}{l}\stackrel{m}{D} \\
\stackrel{2}{N}\end{array}$ & 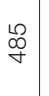 & $\stackrel{8}{8}$ & $\begin{array}{l}\stackrel{c}{o} \\
\stackrel{c}{c}\end{array}$ & 芯 & \begin{tabular}{l}
$\swarrow 0$ \\
0 \\
\multirow{\sigma}{*}{} \\
\end{tabular} & \\
\hline & 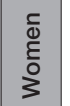 & 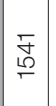 & స్ & 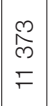 & 0 & 0 & 0 & $\begin{array}{l}5 \\
0 \\
0 \\
b\end{array}$ & $\begin{array}{l}\text { 怘 } \\
\stackrel{\leftrightarrow}{\circ}\end{array}$ & 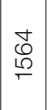 & $\begin{array}{c}\stackrel{\hat{o}}{\mathrm{~N}} \\
\stackrel{\mathrm{N}}{F}\end{array}$ & $\mid \begin{array}{l}\infty \\
\stackrel{\infty}{\infty} \\
\sim \\
\sim\end{array}$ & $\left|\begin{array}{l}0 \\
10 \\
i 0\end{array}\right|$ & 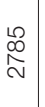 & $\underset{\sim}{\infty}$ & 疍 & 官 & 0 & 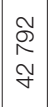 & \\
\hline & 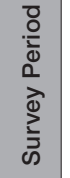 & 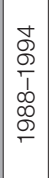 & 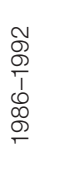 & 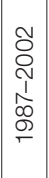 & \begin{tabular}{|l}
0 \\
0 \\
$o$ \\
$T$ \\
\\
\\
\\
\end{tabular} & $\begin{array}{l}0 \\
0 \\
0 \\
1 \\
\\
\\
\\
\end{array}$ & 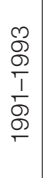 & 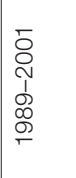 & 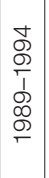 & $\mid \begin{array}{l}0 \\
o \\
o \\
T \\
0 \\
\infty \\
\sigma \\
\sigma\end{array}$ & 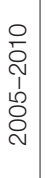 & 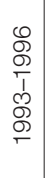 & 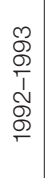 & 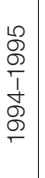 & 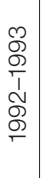 & ભু & 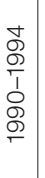 & 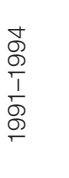 & 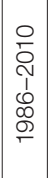 & 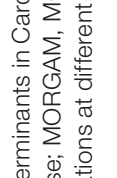 \\
\hline & 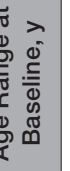 & $\begin{array}{l}0 \\
i \\
o \\
\infty \\
\end{array}$ & 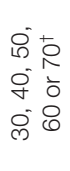 & $\begin{array}{l}\text { d } \\
\hat{1} \\
\dot{m}\end{array}$ & $\mid \begin{array}{l}0 \\
0 \\
1 \\
0 \\
q \\
\end{array}$ & $\begin{array}{l}0 \\
0 \\
⿱ \\
\sigma\end{array}$ & 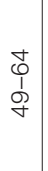 & \begin{tabular}{l}
\multirow{N}{1}{} \\
0 \\
0
\end{tabular} & $\begin{array}{l}0 \\
0 \\
1 \\
0 \\
0\end{array}$ & $\mid \begin{array}{l}\llcorner \\
1 \\
0 \\
0\end{array}$ & \begin{tabular}{l} 
d \\
\multirow{1}{*}{} \\
d
\end{tabular} & $\begin{array}{l}\text { स } \\
\hat{1} \\
0 \\
m\end{array}$ & $\mid \begin{array}{l}\llcorner \\
1 \\
1 \\
m \\
\infty\end{array}$ & 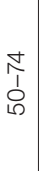 & $\begin{array}{l}0 \\
0 \\
1 \\
d \\
m\end{array}$ & $\begin{array}{l}0 \\
0 \\
0 \\
\text { d } \\
0\end{array}$ & 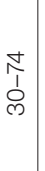 & 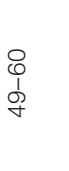 & $\begin{array}{l}\text { t } \\
\hat{D} \\
\dot{m}\end{array}$ & \\
\hline & $\begin{array}{l}\frac{0}{0} \\
\frac{0}{0} \\
\frac{0}{0} \\
0 \\
\dot{0} \\
\dot{0} \\
\dot{2}\end{array}$ & $\sim$ & $\sim$ & $\nabla$ & $\tau$ & $\tau$ & - & $\infty$ & $\sim$ & m & $\tau$ & $\infty$ & - & $\tau$ & $\tau$ & \ulcorner & $\sim$ & $\tau$ & ৪ & \\
\hline & 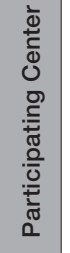 & 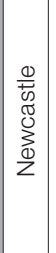 & $\begin{array}{l}\frac{1}{1} \\
\bar{z} \\
0 \\
\sum_{1} \\
\vdots \\
0\end{array}$ & 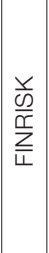 & 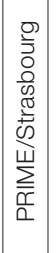 & 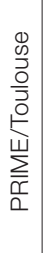 & 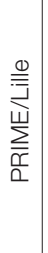 & 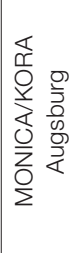 & $\begin{array}{l}\frac{\mathbb{N}}{0} \\
\frac{\tilde{\omega}}{\bar{\omega}} \\
\frac{\bar{m}}{2}\end{array}$ & 彦 & 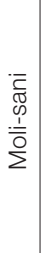 & 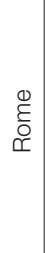 & 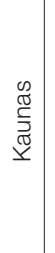 & 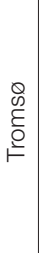 & 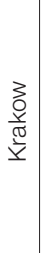 & 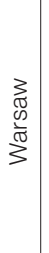 & 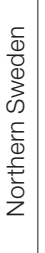 & 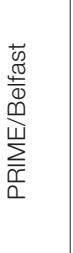 & & \\
\hline & 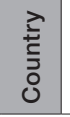 & 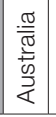 & 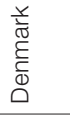 & 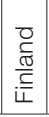 & 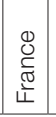 & 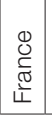 & $\bar{\Psi}$ & 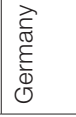 & & $\mid$\begin{tabular}{|l} 
离 \\
\pm
\end{tabular} & & & 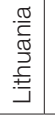 & 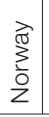 & $\begin{array}{l}\overline{0} \\
\frac{\tilde{\sigma}}{0} \\
0 \\
0\end{array}$ & 0 & \begin{tabular}{l|}
$c$ \\
$\bar{D}$ \\
0 \\
0 \\
0 \\
0
\end{tabular} & 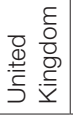 & 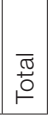 & \\
\hline
\end{tabular}




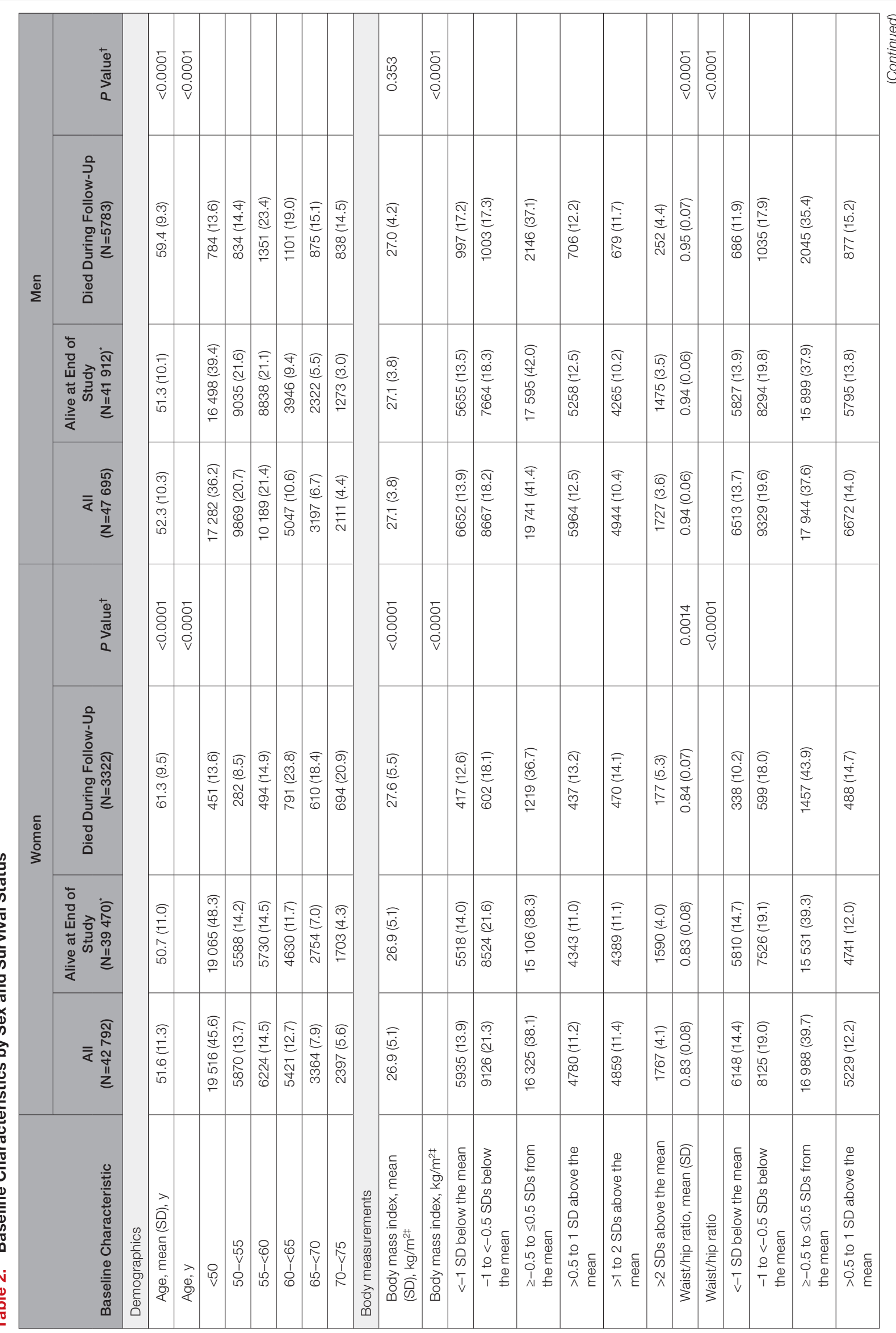




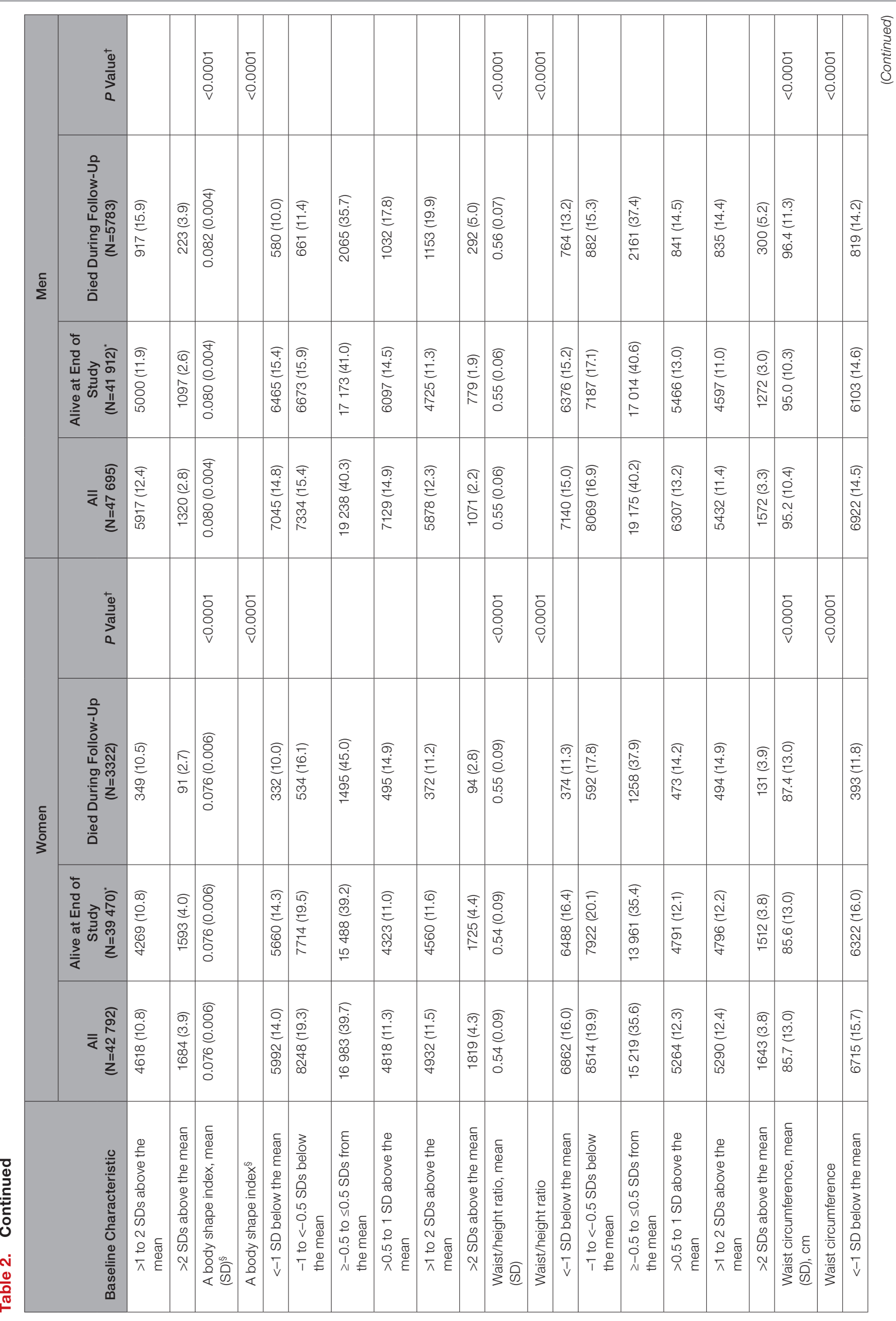




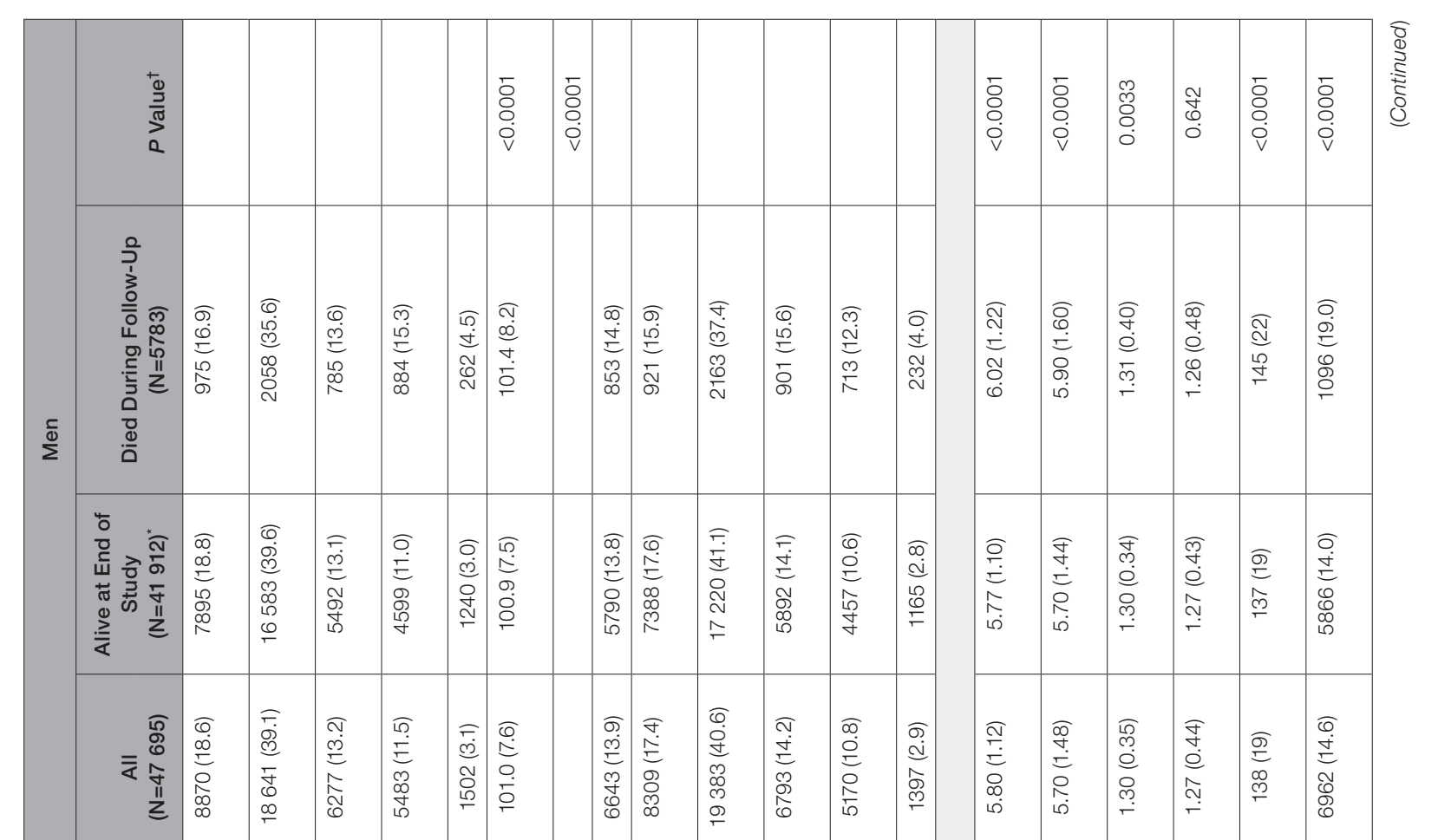

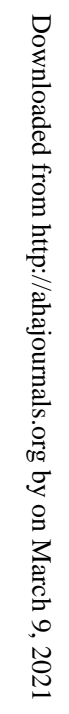

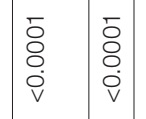

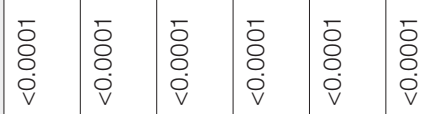

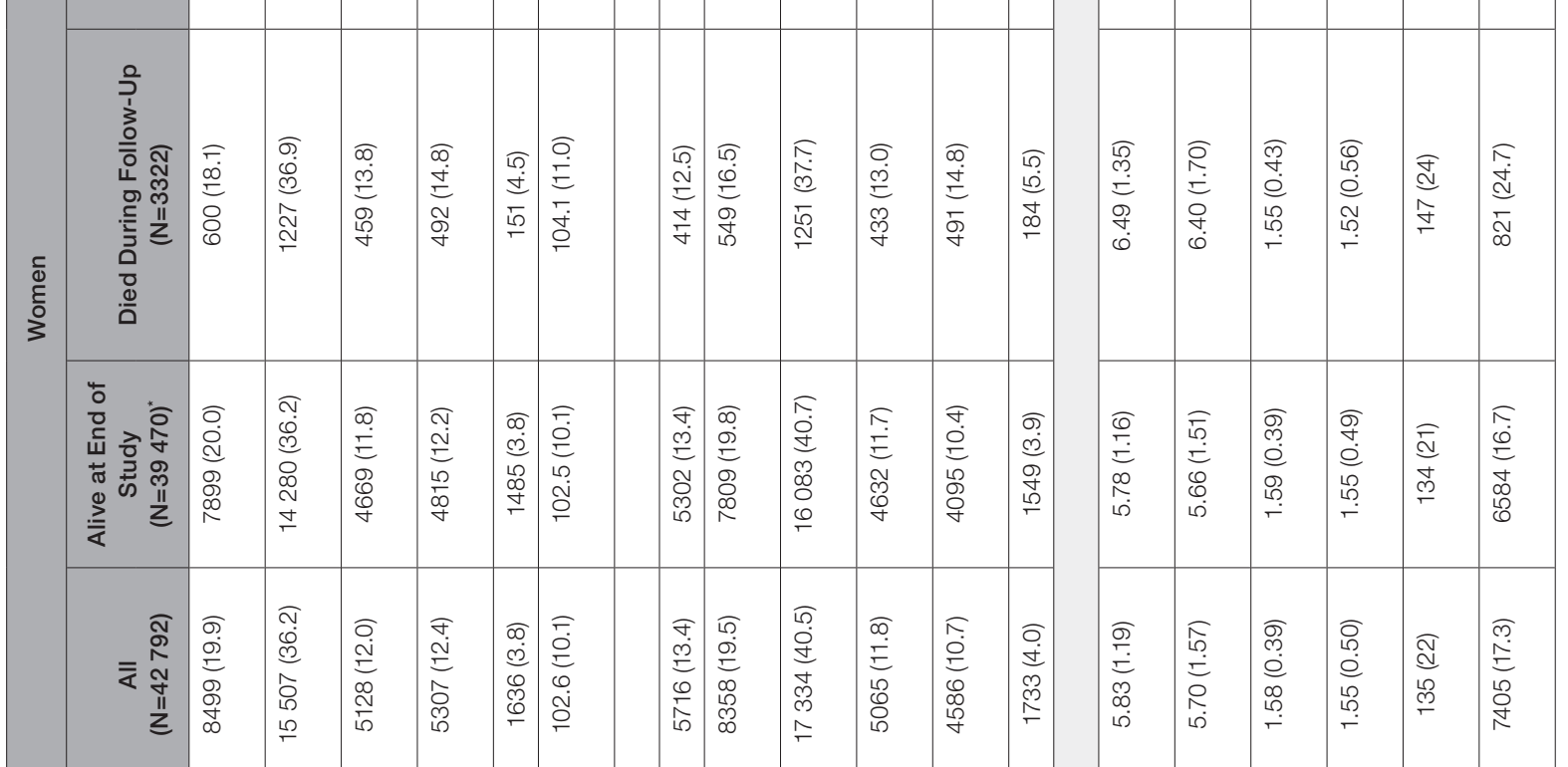




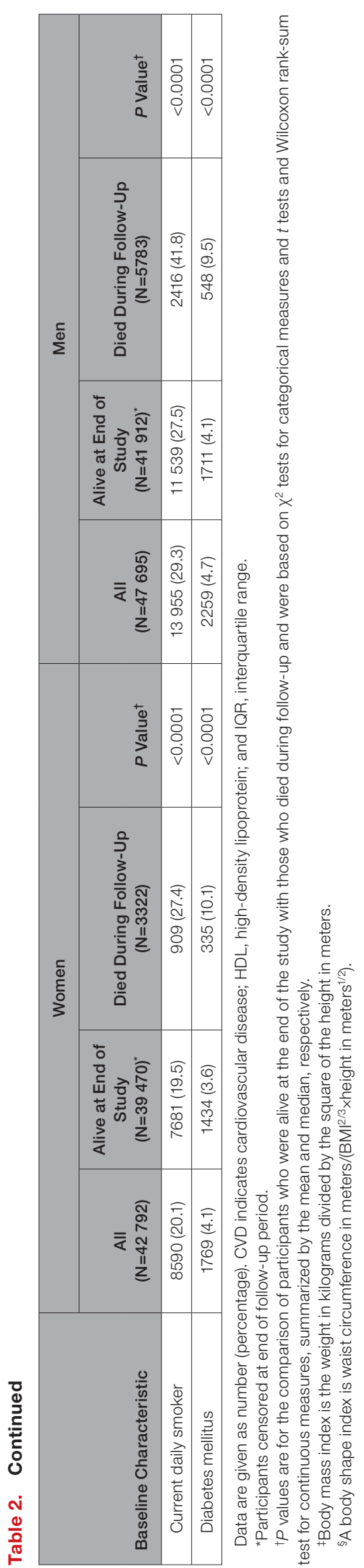

measures used in the analyses. We compared the characteristics of participants included in, and excluded from, the analysis sample (Table S3). We assessed potential collinearity (and interaction) between WC and $\mathrm{HC}$ by creating a single joint measure of WC and $\mathrm{HC}$, with 21 categories for women and 22 categories for men representing all WC and $\mathrm{HC}$ combinations. Some categories were combined to ensure sufficient numbers. The reference category was defined as both $\mathrm{HC}$ and WC being $\geq-0.5$ to $\leq 0.5$ SDs from the mean. For both men and women, estimated hazard ratios for levels of the single joint measure were comparable to those obtained from models including $\mathrm{WC}$ and $\mathrm{HC}$ as separate measures (results not presented). Further sensitivity analyses were performed for all-cause death, (1) excluding those who died $<2$ years from baseline, (2) excluding those reporting diabetes mellitus at baseline, and (3) including an interaction between daily smokers at baseline and each anthropometric measure. All statistical analyses were run using Stata 15.

\section{RESULTS}

\section{Characteristics of Study Participants}

The mean (SD) age of participants was 52.0 (10.8) years, and $52.7 \%$ were men. Anthropometric and other baseline measures are summarized by sex and survival status in Table 2. During a median (interquartile range) follow-up of 10.0 (10.4) years, 9105/90 487 (10.1\%) participants died from all causes, and 2577 (2.8\%) died from cardiovascular causes. Follow-up periods and mortality rates are summarized by participating center and sex in Table 1.

Characteristics of participants included in the analysis and those excluded because of incomplete data were similar (Table S3), except that the analysis sample included a higher proportion of men $(52.7 \%$ versus $44.3 \%)$ and participants aged $<50$ years $(40.7 \%$ versus $31.8 \%)$.

\section{Associations Between Individual Anthropometric Measures and Death}

All anthropometric measures were strongly associated with risk of all-cause and CVD death, for both men and women, in both the partially and further adjusted models (Tables S4 through S7). Estimated adjusted hazard ratios for all-cause and CVD death, for each individual anthropometric measure relative to the reference category ( $\geq-0.5$ to $\leq 0.5$ SDs around mean), are shown by sex in Figure 1.

Further adjusted hazard ratios for all-cause death followed different patterns across levels of the obesity measures. A U-shaped pattern (increased risk for both the lowest and highest levels of the measure) was observed for $\mathrm{BMl}$ and $\mathrm{HC}$ in men and women and for WC 


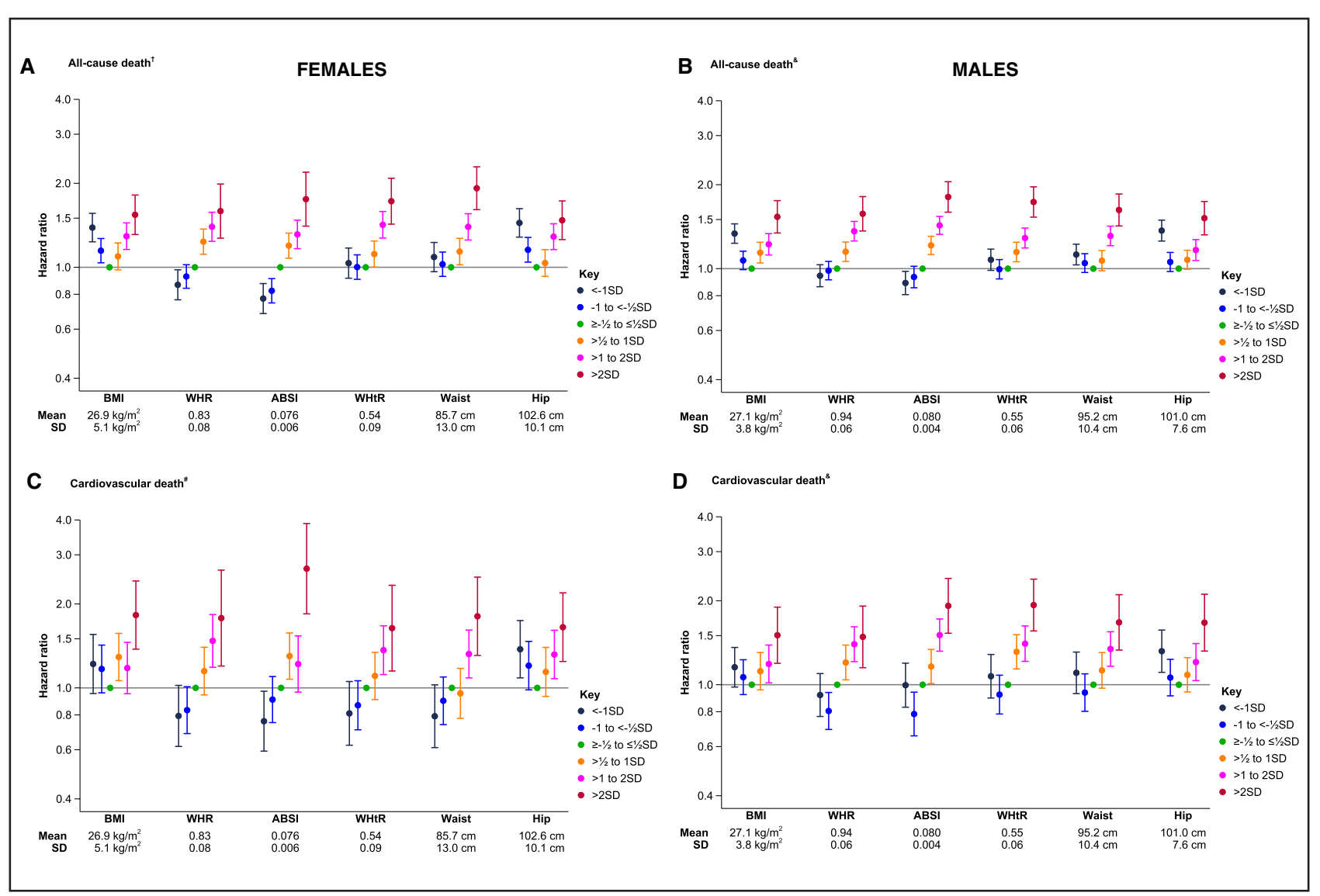

Figure 1. Risk of death, according to obesity measures, by sex and cause of death, estimated after adjustment for cardiovascular risk factors.

Hazard ratios for all-cause (A and B) and cardiovascular (C and D) death by sex, with 95\% Cls, are shown for each obesity measure: body mass index (BMl; weight in kilograms divided by the square of the height in meters), weight/hip ratio (WHR), a body shape index (ABSI; waist circumference in meters/[BMI ${ }^{2 / 3}$ xheight in meters $\left.{ }^{1 / 2}\right]$ ), weight/height ratio (WHtR), waist circumference, and hip circumference. Vertical lines indicate $95 \%$ Cls. Reference category for each obesity measure was $\geq-0.5$ SDs to $\leq 0.5$ SDs from the sample sex-specific mean. Risks were estimated from Cox proportional hazards models, stratified by cohort, and adjusted for age at baseline, log of total cholesterol (mmol/L), log of high-density lipoprotein (HDL) cholesterol (mmol/L), systolic blood pressure (mm Hg), taking antihypertensive drugs, current daily smoker (cigarettes, cigars/cigarillos, or pipe), diabetes mellitus, and all interactions between age and baseline measures that were statistically significant $(P<0.001)$. ${ }^{\dagger}$ Additionally adjusted for an interaction between age at baseline and log of HDL cholesterol (mmol/L). "Additionally adjusted for interaction between age at baseline and systolic blood pressure. ${ }^{\&}$ Additionally adjusted for interaction between age at baseline and current daily smoker.

in men. Risk increased monotonically with $A B S I$ and WHR, with reduced risk for some categories below the mean. A J-shaped association was seen for waist/ height ratio for both men and women and for WC in women.

The further adjusted associations between the anthropometric measures and CVD death followed an essentially similar pattern, with wider Cls for both men and women.

\section{Associations Between WC and HC Measures and Death}

When both WC and $\mathrm{HC}$ were included as predictors of all-cause death in the same partially or further adjusted model (reference category $\geq-0.5$ to $\leq 0.5$ SDs from the mean), both variables remained strongly associated with risk of death $(P<0.0001$; Tables S4 and S5). There was a monotonic relationship between WC and risk of death for both women and men. For women, further adjusted hazard ratios increased from $0.79(95 \% \mathrm{Cl}, 0.68-0.91)$ for $\mathrm{WC}<-1 \mathrm{SD}$ below the mean to $2.16(95 \% \mathrm{Cl}, 1.72-2.71)$ for $\mathrm{WC}>2$ SDs above the mean. For men, the further adjusted hazard ratios increased from $0.90(95 \% \mathrm{Cl}, 0.81-0.99)$ to $1.74(95 \% \mathrm{Cl}, 1.46-2.08)$ in the smallest and largest WC categories, respectively. In the same model, lower $\mathrm{HC}$ categories were associated with increased risk in women $(<-1$ SD below the mean: $1.72[95 \%$ $\mathrm{Cl}, 1.49-1.98] ;-1$ to $<-0.5$ SDs below the mean: 1.27 [95\% Cl, 1.14-1.42]) and men $(<-1$ SD below the mean: 1.52 [95\% Cl, 1.37-1.69]; -1 to $<-0.5$ SDs 


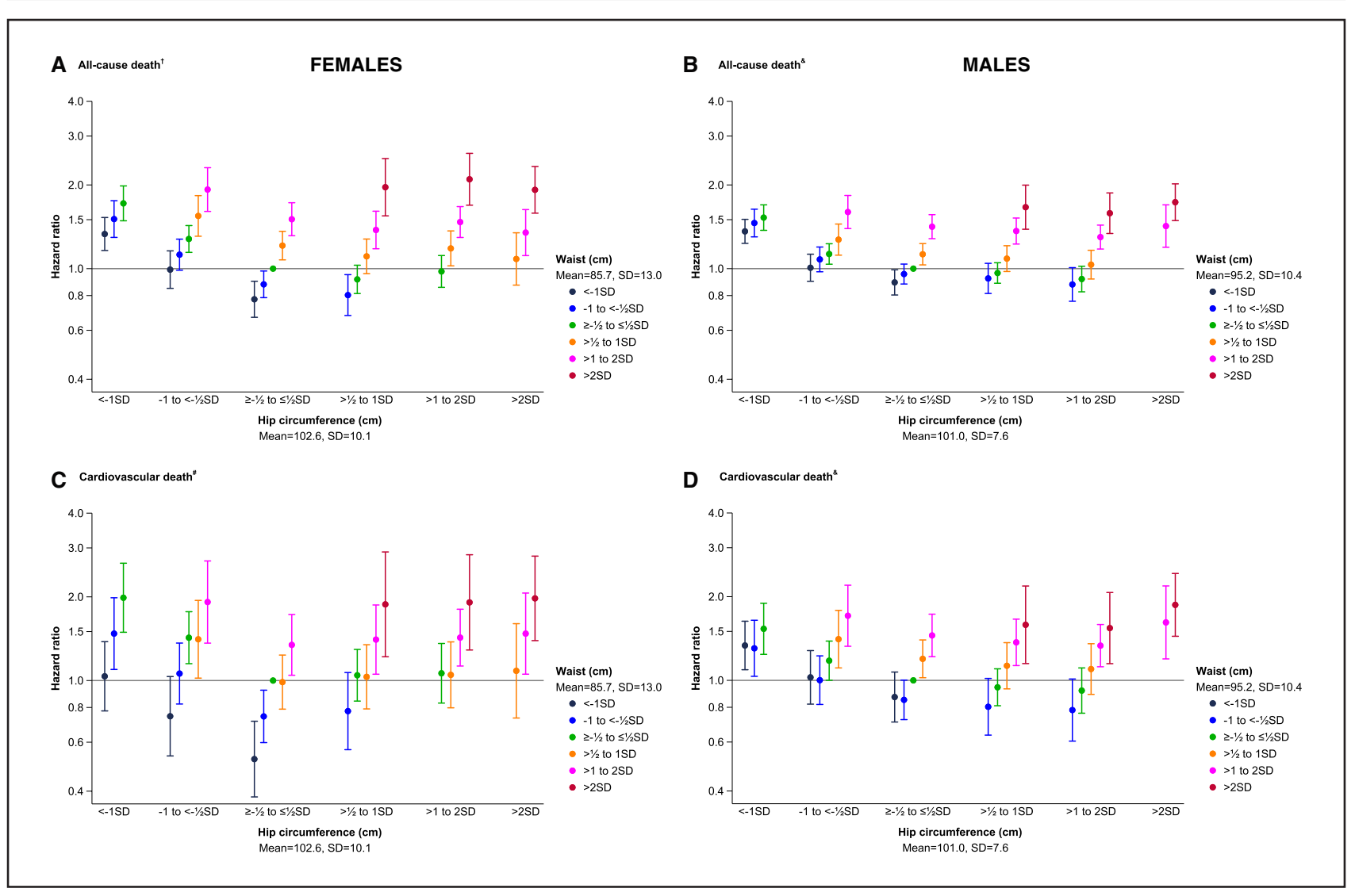

Figure 2. Risk of death, according to waist circumference within hip circumference levels, by sex and cause of death, estimated after adjustment for cardiovascular risk factors.

Hazard ratios for all-cause (A and $\mathbf{B})$ and cardiovascular (C and $\mathbf{D})$ death by sex, with $95 \%$ Cls, are shown for all feasible combinations of waist circumference and hip circumference. Vertical lines indicate $95 \%$ Cls. Reference category was $\geq-0.5$ SDs to $\leq 0.5$ SDs from the sample sex-specific mean for both waist circumference and hip circumference. Risks were estimated from Cox proportional hazards models, stratified by cohort, and adjusted for age at baseline, log of total cholesterol (mmol/L), log of high-density lipoprotein (HDL) cholesterol ( $\mathrm{mmol} / \mathrm{L})$, systolic blood pressure $(\mathrm{mm} \mathrm{Hg})$, taking antihypertensive drugs, current daily smoker (cigarettes, cigars/ cigarillos, or pipe), diabetes mellitus, and all interactions between age and baseline measures that were statistically significant $(P<0.001)$. ${ }^{\dagger}$ Additionally adjusted for an interaction between age at baseline and log of $\mathrm{HDL}$ cholesterol (mmol/L). ${ }^{\#}$ Additionally adjusted for an interaction between age at baseline and systolic blood pressure. ${ }^{\circledR}$ Additionally adjusted for an interaction between age at baseline and current daily smoker.

below the mean: 1.13 [95\% Cl, 1.04-1.23]), with no increased risks for $\mathrm{HC}$ categories above the reference level.

We report the combined influence of $\mathrm{HC}$ and $\mathrm{WC}$ on risk of death in 2 ways, conditional on $\mathrm{HC}$ levels (Figure 2) and conditional on WC levels (Figure 3). Both figures show the estimated adjusted hazard ratios for feasible combinations of $\mathrm{WC}$ and $\mathrm{HC}$ relative to the reference category $(\geq-0.5$ to $\leq 0.5$ SDs from the mean for both WC and $\mathrm{HC}$ ). Figure 2 shows a strong, monotonic relationship between WC and risk of death for all $\mathrm{HC}$ categories, with smaller WC associated with lower risk of death, except for those with the smallest hips $(<-1$ SD below the mean in men). Figure 3 shows the pattern when considering the impact of $\mathrm{HC}$ for different levels of WC. For both men and women with a smaller waist (WC $\leq 1 \mathrm{SD}$ above the mean: $\leq 98.8 \mathrm{~cm}$ in women and $\leq 105.7 \mathrm{~cm}$ in men), larger hips were associated with a reduction in risk. For WC $>1 \mathrm{SD}$ above the mean, risk of death did not change with $\mathrm{HC}$ for men or women.

Similar patterns were seen for CVD death (Tables S6 and S7, Figures 1 through 3).

\section{Sensitivity Analyses}

Estimates were robust to excluding participants who died within 2 years from baseline ( $n=640 ; 0.7 \%)$ or those reporting diabetes mellitus at baseline $(n=4028 ; 4.5 \%)$ (estimates not presented). We assessed period effect; by adding decade of enrollment to the further adjusted models. We found no statistical evidence of a period effect for both men and women (estimates not presented). To investigate 


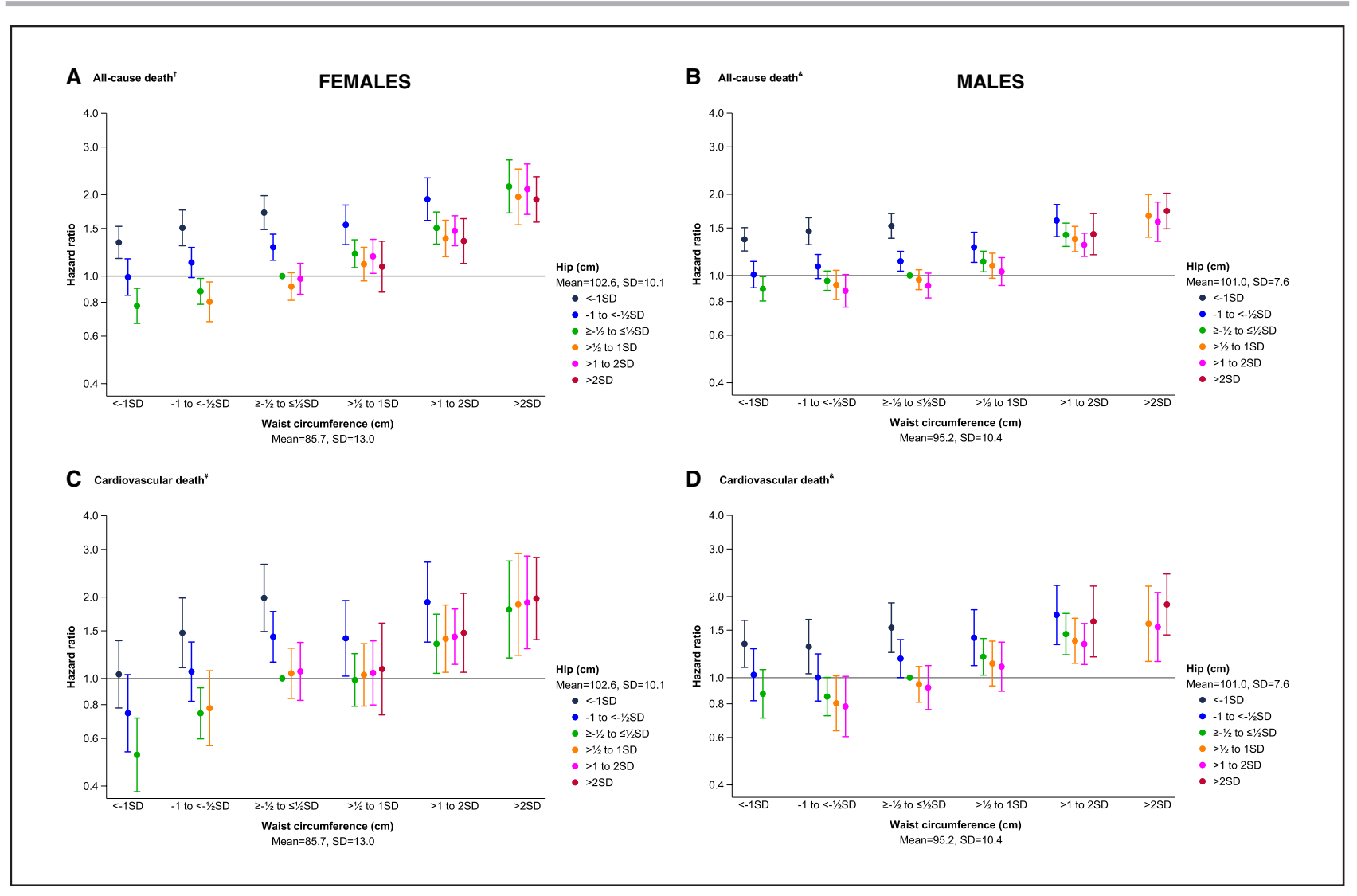

Figure 3. Risk of death, according to hip circumference within waist circumference levels, by sex and cause of death, estimated after adjustment for cardiovascular risk factors.

Hazard ratios for all-cause (A and $\mathbf{B})$ and cardiovascular ( $\mathbf{C}$ and $\mathbf{D})$ death by sex, with $95 \%$ Cls, are shown for all feasible combinations of waist circumference and hip circumference. Vertical lines indicate $95 \%$ Cls. Reference category was $\geq-0.5$ SDs to $\leq 0.5$ SDs from the sample sex-specific mean for both waist circumference and hip circumference. Risks were estimated from Cox proportional hazards models, stratified by cohort, and adjusted for age at baseline, log of total cholesterol (mmol/L), log of high-density lipoprotein $(\mathrm{HDL})$ cholesterol $(\mathrm{mmol} / \mathrm{L})$, systolic blood pressure $(\mathrm{mm} \mathrm{Hg})$, taking antihypertensive drugs, current daily smoker (cigarettes, cigars/ cigarillos, or pipe), diabetes mellitus, and all interactions between age and baseline measures that were statistically significant $(P<0.001)$. ${ }^{\dagger}$ Additionally adjusted for an interaction between age at baseline and log of $\mathrm{HDL}$ cholesterol (mmol/L). ${ }^{*}$ Additionally adjusted for an interaction between age at baseline and systolic blood pressure. \&Additionally adjusted for an interaction between age at baseline and current daily smoker.

whether the $\mathrm{U}$ - or J-shaped relationship between mortality and some of the anthropometric measures could be explained by mortality due to diseases associated with smoking (which also affect anthropometric measures), we fitted models that additionally included baseline smoking status and the interaction between smoking and anthropometric measures. The adjusted estimates for nonsmokers and smokers are presented in Figure S1. Because the main aim of this analysis was to understand whether the shape of the association for the anthropometric categories was similar for smokers and nonsmokers, the hazard ratio estimates presented for smokers did not include the risk associated with smoking (ie, risk was estimated using main effect of anthropometric measure plus the interaction term between smoking and the anthropometric measure). The shapes of the relationships between the body measures and risk of death were similar.

\section{DISCUSSION}

This study of $>90000$ predominantly white adults followed up for up to 24 years provides evidence that it is important to consider measures of both upper- and lower-body adiposity when estimating risk of death. The most commonly used measures of obesity (BMI and $\mathrm{WC}$ ) as well as $\mathrm{HC}$ displayed $\mathrm{U}$ - or J-shaped associations with risk of death from all causes. When both WC and HC were included together in models, a more nuanced and clearer picture was revealed, with 2 distinct clinical messages. First, WC was strongly positively associated with risk of death at all hip sizes, except among those with the smallest hips (who almost all have WC well below the mean). In addition, among those with smaller waists, hip size was strongly negatively associated with risk of death. Among the people with smaller waists, who would not normally be identified as being at higher risk of death, having 
smaller hips was clearly associated with increased risk of death. In our sample, by considering both WC and $\mathrm{HC}$, rather than just WC, an additional 19\% of women and $18 \%$ of men would be identified as being at higher risk of death. These results demonstrate the value of considering body shape using both $\mathrm{WC}$ and $\mathrm{HC}$, and contribute to the growing literature on the impact of body fat depots in different parts of the body on risk of death, as well as the evidence for the protective effects of fat-free mass. ${ }^{2,4,6,8,11}$

Like others ${ }^{10}$, we found that $A B S I$ was a strong predictor of death. This suggests that ABSI could be useful in the research setting, particularly because it is a single measure of obesity. It may also have some value in clinical settings if simple calculators based on population norms were created. From a public health perspective, $\mathrm{ABSI}$ is likely to be less useful because of the inherent challenges in communicating messages about risk of death using a highly complex concept and measure.

Our findings add to the small but growing literature examining the combined effect of $\mathrm{WC}$ and $\mathrm{HC}$ as predictors of death. A 2013 literature review on the topic found 5 studies that all suggested the strength of associations between WC and death is underestimated without also considering HC. ${ }^{7}$ Compared with these studies (and one published since), ${ }^{26}$ our study used a larger, multicountry cohort with longer follow-up and a substantial number of deaths. Hazard ratios for combined categories of WC and $\mathrm{HC}$ have only previously been estimated in our earlier study among South Asian and African Mauritians. ${ }^{27}$ The current finding that larger $\mathrm{HC}$ is protective only for people with smaller WC agrees with evidence from that Mauritian study. The stronger relationship between $\mathrm{WC}$ and $\mathrm{HC}$ and risk of death observed in Mauritius may be due to the considerably lower WCs observed in that population (median [interquartile range] WC, 90.5 [17.0] cm for survivors and 94.0 [17.0] cm for those who died from CVD in this sample compared with 79.5 [15.2] $\mathrm{cm}$ for survivors and 84.0 [15.5] cm for those who died from CVD in Mauritius). ${ }^{27}$ It is possible that in the many populations worldwide that currently have a larger mean WC than observed in the historic MORGAM cohort, the protective effect of $\mathrm{HC}$ may be less pronounced. Further studies among populations with different prevalence of obesity at baseline will be important to confirm this hypothesis. Unfortunately, heterogeneity of findings across participating cohorts could not be tested within our analytical framework because of the small number of deaths in some, and different survey periods and age profiles.

Although the physiological basis for the opposing effects of WC and $\mathrm{HC}$ is not completely clear, some hypotheses for the protective effects of lower-body gluteofemoral adipose tissue, and the particularly detrimental effects of visceral adipose tissue, have been put forward. ${ }^{11,12}$ These include clear differences in the ability of the cells in different depots to store and release lipids and the secretion of proteins, such as leptin and adiponectin (positively associated), as well as inflammatory cytokines (negatively associated). ${ }^{11}$ In addition to these metabolic differences, lower-body anthropometric measures are also capturing fat-free (muscle) mass in the thigh and buttocks. Greater muscle mass is likely to be indicative of greater levels of physical activity and less time sedentary, which are both protective against premature death, ${ }^{28,29}$ whereas smaller hips could reflect overall smaller frame size or result from health conditions influenced by lifestyle behaviors. More important, although body shape changes with age, the associations observed were robust across all age groups (up to age 75 years). An extensive literature exists on the association between $\mathrm{HC}$ or thigh circumference (relative to $\mathrm{WC}$ ) and a range of metabolic risk markers and disease outcomes, including dyslipidemia, hypertension, and diabetes mellitus, ${ }^{2-4}$ in addition to the all-cause and CVD mortality observed here.

It is possible that strength training interventions that increase muscle in the hips or thighs without also increasing waist may reduce risk in addition to interventions that target reduction in WC. Although that hypothesis is untested, a Scandinavian study showed no reduction in risk of death among those who reduced their $\mathrm{HC}$ over time. That study, however, did not specifically assess the corresponding changes in WC for those who reduced their $\mathrm{HC} .{ }^{26} \mathrm{~A}$ Danish study found that the risk of death from small hips was partially attenuated by physical activity. ${ }^{30}$

Strengths of this study include the large sample size from 30 cohorts in 11 countries, the consistent methods for measurement of baseline factors and ascertainment of outcomes, and that findings were robust across multiple sensitivity analyses. Limitations include the inability to exclude participants with a diagnosis of cancer, respiratory disease, or abdominal distension (not measured) or pregnancy (recorded in $<50 \%$ of cohorts), the absence of information on potential confounders, such as physical activity and sedentary behavior, the self-report of diabetes mellitus history, and the low incidence of CVD death observed. Furthermore, as all measures were collected at baseline, we were unable to investigate the variation in body measures and CVD risk factors over time and the relationship with the risk of death.

In conclusion, this study provides evidence that a more complex relationship between $\mathrm{HC}, \mathrm{WC}$, and risk of death is revealed when both measures are considered simultaneously. Considering both WC and $\mathrm{HC}$ in the clinical setting could help best identify those at increased risk of death. 


\section{ARTICLE INFORMATION}

Received November 12, 2019; accepted May 6, 2020.

\section{Affiliations}

From the Global Obesity Centre, Institute for Health Transformation (A.J.C.), and Biostatistics Unit, Faculty of Health (H.R., L.O.), Deakin University, Geelong, Australia; Unité d'Epidémiologie et de Santé Publique, Inserm-U1167, Institut Pasteur de Lille, Lille, France (J.D.); School of Public Health, University of Queensland, Herston, Queensland, Australia (A.J.D.); Department of Epidemiology CVD Prevention and Health Promotion, Cardinal Wyszynski National Institute of Cardiology, Warsaw, Poland (W.D.); Department of Medicine and Surgery, Research Center in Epidemiology and Preventive Medicine, University of Insubria, Varese, Italy (M.F., F.G., L.I., G.V.); Department of Cardiology, Toulouse University School of Medicine, Toulouse, France (J.F.); Department of Cardiovascular, Endocrine-Metabolic Diseases and Aging, Istituto Superiore di Sanità, Rome, Italy (S.G., L.P.); University of Insubria, Varese, Italy (F.G., L.I.); Mediterranea Cardiocentro, Napoli, Italy (F.G.); Department of Epidemiology and Prevention, Istituto di Ricovero e Cura a Carattere Scientifico Neuromed, Pozzilli, Italy (L.I.); Department of Public Health Solutions, Finnish Institute for Health and Welfare, Helsinki, Finland (P.J., T.J.N., T.P., M.S., S.V., K.K.); Centre for Public Health, Institute for Health Sciences, Queen's University, School of Medicine, Dentistry and Biomedical Sciences, Belfast, Northern Ireland (F.K.); Department of Public Health, University Hospital of Strasbourg, France (M.M.); Department of Epidemiology and Public Health, University of Strasbourg, France (M.M.); Department of Medicine, Turku University Hospital and University of Turku, Turku, Finland (T.J.N.); Department of Epidemiology and Population Studies, Jagiellonian University Medical College, Kraków, Poland (A.P.); Department of Population Studies, Institute of Cardiology, Lithuanian University of Health Sciences, Kaunas, Lithuania (A.T.); German Research Center for Environmental Health, Institute of Epidemiology, Helmholtz Zentrum München, Neuherberg, Germany (B.T.); Center for Clinical Research and Prevention, Bispebjerg and Frederiksberg Hospital, Copenhagen, Denmark (U.T.); Cardiovascular Prevention Centre, Udine, Italy (D.V.); Department of Community Medicine, The Arctic University of Norway, Tromsø, Norway (T.W.); and Department of Public Health and Clinical Medicine, and Heart Centre, Umeå University, Umeå, Sweden (S.S.).

\section{Acknowledgments}

Drs Cameron, Romaniuk, Orellana, and Söderberg were responsible for the conception and design of the work. Drs Cameron, Romaniuk, and Orellana were responsible for analysis of the data and initial drafting of the manuscript. All authors other than Drs Cameron, Romaniuk, and Orellana were responsible for acquisition of data. All authors were involved in revisions of the manuscript and interpretation of data and have approved the submitted version.

\section{Sources of Funding}

This work was supported by the Medical Research Council London (grant G0601463 [80983]) and European Union FP7 project BiomarCaRE (grant HEALTH-F2-2011-278913), which supported the Monica Risk, Genetics, Archiving, and Monograph (MORGAM) Project. The activities of the MORGAM Data Centre have been sustained also by recent funding from European Union FP7 project Consortium on Health and Ageing: Network of Cohorts in Europe and the United States (HEALTH-F3-2010-242244). Dr Cameron is the recipient of an Australian Research Council Discovery Early Career Researcher Award (grant DE160100141). Dr Söderberg was supported by the Swedish Heart-Lung Foundation, the County Council of Västerbotten (grant ALF, VLL-548791), and Umeå University. All funders had no involvement in the study design; in the collection, analysis, and interpretation of data; in the writing of the report; and in the decision to submit the article for publication. All researchers are independent of the funders and have had full access to all the statistical reports and tables in the study and can take responsibility for the integrity of the data and the accuracy of the data analysis. We are not aware of any conflicts of interest in relation to this work.

\section{Disclosures}

None.

Supplementary Materials
Tables S1-S7
Figure S1

\section{REFERENCES}

1. Global Burden of Disease Obesity Collaborators, Afshin A, Forouzanfar MH, Reitsma MB, Sur P, Estep K, Lee A, Marczak L, Mokdad AH, Moradi-Lakeh M, Naghavi $\mathrm{M}$, et al. Health effects of overweight and obesity in 195 countries over 25 years. N Engl J Med. 2017;377:13-27.

2. Snijder MB, Zimmet PZ, Visser M, Dekker JM, Seidell JC, Shaw JE. Independent and opposite associations of waist and hip circumferences with diabetes, hypertension and dyslipidemia: the AusDiab Study. Int J Obes Relat Metab Disord. 2004;28:402-409.

3. Parker ED, Pereira MA, Stevens J, Folsom AR. Association of hip circumference with incident diabetes and coronary heart disease: the Atherosclerosis Risk in Communities study. Am J Epidemiol. 2009;169:837-847.

4. Canoy D, Boekholdt SM, Wareham N, Luben R, Welch A, Bingham S, Buchan I, Day N, Khaw K-T. Body fat distribution and risk of coronary heart disease in men and women in the European Prospective Investigation Into Cancer and Nutrition in Norfolk cohort: a populationbased prospective study. Circulation. 2007;116:2933-2943.

5. Borges MC, Oliveira IO, Freitas DF, Horta BK, Ong KK, Gigante DP, Barros AJD. Obesity-induced hypoadiponectinaemia: the opposite influences of central and peripheral fat compartments. Int J Epidemiol. 2017:46:2044-2055.

6. Bigaard J, Frederiksen K, Tjonneland A, Thomsen BL, Overvad K, Heitmann BL, Sorensen TIA. Waist and hip circumferences and allcause mortality: usefulness of the waist-to-hip ratio? Int J Obes Relat Metab Disord. 2004;28:741-747.

7. Cameron AJ, Magliano DJ, Söderberg S. A systematic review of the impact of including both waist and hip circumference in risk models for cardiovascular diseases, diabetes and mortality. Obes Rev. 2013;14:86-94.

8. Heitmann BL, Frederiksen P. Thigh circumference and risk of heart disease and premature death: prospective cohort study. $\mathrm{Br}$ Med J. 2009;339:b3292.

9. Neeland IJ, Poirier P, Despres JP. Cardiovascular and metabolic heterogeneity of obesity: clinical challenges and implications for management. Circulation. 2018;137:1391-1406.

10. Krakauer NY, Krakauer JC. A new body shape index predicts mortality hazard independently of body mass index. PLoS One. 2012;7:e39504.

11. Manolopoulos KN, Karpe F, Frayn KN. Gluteofemoral body fat as a determinant of metabolic health. Int J Obes (Lond). 2010;34:949-959.

12. Piche ME, Vasan SK, Hodson L, Karpe F. Relevance of human fat distribution on lipid and lipoprotein metabolism and cardiovascular disease risk. Curr Opin Lipidol. 2018;29:285-292.

13. Larsson B, Svardsudd K, Welin L, Wilhelmsen L, Bjorntorp P, Tibblin G. Abdominal adipose tissue distribution, obesity, and risk of cardiovascular disease and death: 13 year follow up of participants in the study of men born in 1913. Br Med J (Clin Res Ed). 1984;288:1401-1404.

14. Ashwell M, Gunn P, Gibson S. Waist-to-height ratio is a better screening tool than waist circumference and BMI for adult cardiometabolic risk factors: systematic review and meta-analysis. Obes Rev. 2012;13:275-286.

15. Ashwell M, Lejeune S, McPherson K. Ratio of waist circumference to height may be better indicator of need for weight management. Br Med J. 1996;312:377.

16. Allison DB, Paultre F, Goran MI, PoehIman ET, Heymsfield SB. Statistical considerations regarding the use of ratios to adjust data. Int $J$ Obes Relat Metab Disord. 1995;19:644-652.

17. Molarius A, Seidell JC, Sans S, Tuomilehto J, Kuulasmaa K. Waist and hip circumferences, and waist-hip ratio in 19 populations of the WHO MONICA Project. Int J Obes Relat Metab Disord. 1999;23:116-125.

18. Pischon T, Boeing H, Hoffmann K, Bergmann M, Schulze MB, Overvad K, van der Schouw YT, Spencer E, Moons KGM, Tjønneland A, et al. General and abdominal adiposity and risk of death in Europe. N Engl $J$ Med. 2008;359:2105-2120.

19. Flegal KM, Graubard BI. Estimates of excess deaths associated with body mass index and other anthropometric variables. Am J Clin Nutr. 2009;89:1213-1219.

20. Evans A, Salomaa V, Kulathinal S, Asplund K, Cambien F, Ferrario M, Perola M, Peltonen L, Shields D, Tunstall-Pedoeet $H$, et al. MORGAM (an international pooling of cardiovascular cohorts). Int J Epidemiol. 2005;34:21-27.

21. MORGAM Project. Description and quality assessment of MORGAM data. 2008. https://thl.fi/publications/morgam/qa/baseline/contents. $\mathrm{htm}$. Accessed March 13, 2019.

22. Kulathinal S, Niemelä M, Niiranen T, Saarela O, Palosaari T, Tapanainen $\mathrm{H}$, Kuulasmaa K. Description of MORGAM cohorts. MORGAM Project 
e-publications [Internet]. 2005. (2). URN:NBN:fi-fe20051214. https://thl. fi/publications/morgam/cohorts/index.html. Accessed April 6, 2020.

23. World Health Organization (WHO). WHO MONICA Project e-publications (ISSN 2242-1246), No. 1.5. 1999. http://www.thl.fi/publications/monica/ manual/part1/i-2.htm. URN:NBN:fi-fe19981148. Accessed March 13, 2019

24. MORGAM Project. MORGAM manual. MORGAM Project e-publications [Internet]. 2001; (1). URN:NBN:fi-fe20041529. https://thl.fi/publi cations/morgam/manual/contents.htm. Accessed April 6, 2020.

25. D'Agostino RB Sr, Vasan RS, Pencina MJ, Wolf PA, Cobain M, Massaro JM, Kannel WB. General cardiovascular risk profile for use in primary care: the Framingham Heart Study. Circulation. 2008;117:743-753.

26. Lanfer A, Mehlig K, Heitmann BL, Lissner L. Does change in hip circumference predict cardiovascular disease and overall mortality in Danish and Swedish women? Obesity (Silver Spring). 2014;22: 957-963.
27. Cameron AJ, Magliano DJ, Shaw JE, Zimmet PZ, Carstensen B, Albert KG, Tuomilehto J, Barr EL, Pauvaday VK, Kowlessur S, et al. The influence of hip circumference on the relationship between abdominal obesity and mortality. Int J Epidemiol. 2012;41:484-494.

28. Dunstan DW, Barr EL, Healy GN, Salmon J, Shaw JE, Balkau B, Magliano DJ, Cameron AJ, Zimmet PZ, Owen N. Television viewing time and mortality: the Australian Diabetes, Obesity and Lifestyle Study (AusDiab). Circulation. 2010;121:384-391.

29. Bellavia A, Bottai M, Wolk A, Orsini N. Physical activity and mortality in a prospective cohort of middle-aged and elderly men-a time perspective. Int J Behav Nutr Phys Act. 2013;10:94.

30. Ostergaard JN, Gronbaek M, Angquist L, Schnohr P, Sorensen TI, Heitmann BL. Combined influence of leisure-time physical activity and hip circumference on all-cause mortality. Obesity (Silver Spring). 2013;21:E78-E85 


\section{Supplemental Material}


Table S1. Range of values for obesity measure categories by sex .

\begin{tabular}{|c|c|c|c|c|c|c|}
\hline \multirow[b]{2}{*}{ Obesity Measure } & \multicolumn{6}{|c|}{ CATEGORY } \\
\hline & $\begin{array}{c}<-1 \mathrm{SD} \\
\text { below the mean }\end{array}$ & $\begin{array}{c}-1 \text { to }<-1 / 2 \mathrm{SD} \\
\text { below the mean }\end{array}$ & $\begin{array}{c}\geq-1 / 2 \text { to } \leq 1 / 2 \text { SD } \\
\text { from the mean }\end{array}$ & $\begin{array}{c}>1 / 2 \text { to } 1 \mathrm{SD} \\
\text { above the mean }\end{array}$ & $\begin{array}{c}>1 \text { to } 2 \mathrm{SD} \\
\text { above the mean }\end{array}$ & $\begin{array}{c}>2 \mathrm{SD} \\
\text { above the mean }\end{array}$ \\
\hline \multicolumn{7}{|c|}{ Body mass index $\left(\mathrm{kg} / \mathrm{m}^{2}\right)^{+}$} \\
\hline Females & $<21.8$ & $21.8-<24.3$ & $24.3-29.4$ & $>29.4-32.0$ & $>32.0-37.1$ & $>37.1$ \\
\hline Males & $<23.3$ & $23.3-<25.2$ & $25.2-29.0$ & $>29.0-30.9$ & $>30.9-34.8$ & $>34.8$ \\
\hline \multicolumn{7}{|l|}{ Waist-to-hip ratio } \\
\hline Females & $<0.76$ & $0.76-<0.80$ & $0.80-0.87$ & $>0.87-0.91$ & $>0.91-0.99$ & $>0.99$ \\
\hline Males & $<0.88$ & $0.88-<0.91$ & $0.91-0.97$ & $>0.97-1.01$ & $>1.01-1.07$ & $>1.07$ \\
\hline \multicolumn{7}{|c|}{ A body shape index $\dagger$} \\
\hline Females & $<0.070$ & $0.070-<0.073$ & $0.073-0.079$ & $>0.079-0.082$ & $>0.082-0.088$ & $>0.088$ \\
\hline Males & $<0.076$ & $0.076-<0.078$ & $0.078-0.083$ & $>0.083-0.085$ & $>0.085-0.089$ & $>0.089$ \\
\hline \multicolumn{7}{|c|}{ Waist-to-height ratio } \\
\hline Females & $<0.45$ & $0.45-<0.49$ & $0.49-0.58$ & $>0.58-0.63$ & $>0.63-0.71$ & $>0.71$ \\
\hline Males & $<0.49$ & $0.49-<0.52$ & $0.52-0.58$ & $>0.58-0.61$ & $>0.61-0.68$ & $>0.68$ \\
\hline \multicolumn{7}{|c|}{ Waist circumference (cm) } \\
\hline Females & $<72.8$ & $72.8-<79.3$ & $79.3-92.3$ & $>92.3-98.8$ & $>98.8-111.8$ & $>111.8$ \\
\hline Males & $<84.8$ & $84.8-<90.0$ & $90.0-100.5$ & $>100.5-105.7$ & $>105.7-116.2$ & $>116.2$ \\
\hline \multicolumn{7}{|c|}{ Hip circumference (cm) } \\
\hline Females & $<92.5$ & $92.5-<97.6$ & $97.6-107.7$ & $>107.7-112.8$ & $>112.8-123.0$ & $>123.0$ \\
\hline Males & $<93.3$ & $93.3-<97.2$ & $97.2-104.8$ & $>104.8-108.6$ & $>108.6-116.2$ & $>116.2$ \\
\hline
\end{tabular}

Body-mass index (BMI) is the weight in kilograms divided by the square of the height in meters.

$\dagger$ A body shape index is waist circumference in metres/(BMI ${ }^{2 / 3} *$ height in meters $\left.{ }^{1 / 2}\right)$. 
Table S2. Number and proportion of participants in each combination of hip and waist circumference categories, by sex.

\begin{tabular}{|c|c|c|c|c|c|c|c|c|c|c|c|c|}
\hline \multirow[b]{2}{*}{ Waist circumference } & \multicolumn{12}{|c|}{ HIP CIRCUMFERENCE } \\
\hline & \multicolumn{2}{|c|}{$\begin{array}{c}<-1 S D \\
\text { below the mean } \\
n \quad(\%)\end{array}$} & \multicolumn{2}{|c|}{$\begin{array}{c}-1 \text { to }<-1 / 2 \text { SD } \\
\text { below the mean }\end{array}$} & \multicolumn{2}{|c|}{$\begin{array}{c}\geq-1 / 2 \text { to } \leq 1 / 2 \text { SD } \\
\text { from the mean }\end{array}$} & \multicolumn{2}{|c|}{$\begin{array}{c}>1 / 2 \text { to } 1 S D \\
\text { above the mean }\end{array}$} & \multicolumn{2}{|c|}{$\begin{array}{c}>1 \text { to } 2 S D \\
\text { above the mean }\end{array}$} & \multicolumn{2}{|c|}{$\begin{array}{l}\qquad 2 \mathrm{SD} \\
\text { above the mean }\end{array}$} \\
\hline FEMALES $(\mathrm{N}=42,792)$ & & & & & & & & & & & & \\
\hline$<-1 \mathrm{SD}$ below the mean & 3,528 & $(8.2)$ & 2,282 & $(5.3)$ & 880 & $(2.1)$ & 23 & $(0.054)$ & 2 & $(0.0047)$ & 0 & $(0)$ \\
\hline-1 to $<-1 / 2 \mathrm{SD}$ below the mean & 1,508 & $(3.5)$ & 3,344 & $(7.8)$ & 3,486 & $(8.1)$ & 142 & $(0.33)$ & 18 & $(0.042)$ & 1 & $(0.0023)$ \\
\hline$\geq-1 / 2$ to $\leq 1 / 2 \mathrm{SD}$ from the mean & 623 & $(1.5)$ & 2,443 & $(5.7)$ & 9,548 & $(22)$ & 2,163 & $(5.1)$ & 709 & $(1.7)$ & 21 & $(0.049)$ \\
\hline$>1 / 2$ to $1 \mathrm{SD}$ above the mean & 33 & $(0.077)$ & 207 & $(0.48)$ & 2,136 & $(5.0)$ & 1,406 & (3.3) & 1,245 & (2.9) & 101 & $(0.24)$ \\
\hline$>1$ to $2 \mathrm{SD}$ above the mean & 23 & $(0.054)$ & 75 & $(0.18)$ & 1,217 & $(2.8)$ & 1,206 & $(2.8)$ & 2,060 & $(4.8)$ & 726 & $(1.7)$ \\
\hline$>2 \mathrm{SD}$ above the mean & 1 & $(0.0023)$ & 7 & $(0.016)$ & 67 & $(0.16)$ & 125 & $(0.29)$ & 552 & (1.3) & 884 & $(2.1)$ \\
\hline MALES $(\mathrm{N}=\mathbf{4 7 , 6 9 5 )}$ & & & & & & & & & & & & \\
\hline$<-1 \mathrm{SD}$ below the mean & 3,718 & $(7.8)$ & 2,054 & (4.3) & 1,115 & $(2.3)$ & 30 & $(0.063)$ & 5 & $(0.010)$ & 0 & $(0)$ \\
\hline-1 to $<-1 / 2 \mathrm{SD}$ below the mean & 1,910 & $(4.0)$ & 2,964 & $(6.2)$ & 3,718 & $(7.8)$ & 226 & $(0.47)$ & 50 & $(0.10)$ & 2 & $(0.0042)$ \\
\hline$\geq-1 / 2$ to $\leq 1 / 2 \mathrm{SD}$ from the mean & 965 & $(2.0)$ & 3,034 & $(6.4)$ & 11,020 & (23) & 2,852 & $(6.0)$ & 747 & $(1.6)$ & 23 & $(0.048)$ \\
\hline$>1 / 2$ to $1 \mathrm{SD}$ above the mean & 39 & $(0.082)$ & 207 & $(0.43)$ & 2,587 & $(5.4)$ & 2,120 & $(4.4)$ & 1,285 & $(2.7)$ & 39 & $(0.082)$ \\
\hline$>1$ to $2 \mathrm{SD}$ above the mean & 11 & $(0.023)$ & 49 & $(0.10)$ & 906 & $(1.9)$ & 1,478 & $(3.1)$ & 2,577 & (5.4) & 462 & $(1.0)$ \\
\hline$>2 \mathrm{SD}$ above the mean & 0 & $(0)$ & 1 & $(0.0021)$ & 37 & $(0.078)$ & 87 & $(0.18)$ & 506 & $(1.1)$ & 871 & $(1.8)$ \\
\hline
\end{tabular}


Table S3. Summary of follow-up times, mortality rates and baseline characteristics of participants included in and excluded from the analysis sample.

\begin{tabular}{|c|c|c|c|c|c|c|}
\hline \multirow[t]{2}{*}{ Characteristic } & \multicolumn{2}{|c|}{$\begin{array}{c}\text { Analysis } \\
\text { Sample } \\
(\mathrm{N}=90,487)\end{array}$} & \multicolumn{4}{|c|}{$\begin{array}{c}\text { Excluded from analysis } \\
\text { sample }^{\$} \\
(\mathrm{~N}=6,702)\end{array}$} \\
\hline & $\mathbf{n}$ & $(\%)$ & $\mathbf{N}^{¥}$ & $(\%)^{\#}$ & $\mathbf{n}$ & $(\%)$ \\
\hline \multicolumn{7}{|l|}{ Survival } \\
\hline All-cause deaths & 9,105 & $(10.1)$ & 6,702 & $(100)$ & 777 & $(11.6)$ \\
\hline CVD deaths & 2,577 & $(2.8)$ & 6,702 & $(100)$ & 241 & $(3.6)$ \\
\hline Median (IQR) follow-up (years) & 10.0 & $(10.4)$ & 6,702 & $(100)$ & 9.5 & $(6.8)$ \\
\hline Mean (SD) age died/censored (years) & 63.1 & $(11.4)$ & 6,702 & $(100)$ & 63.6 & $(11.1)$ \\
\hline \multicolumn{7}{|l|}{ Demographics } \\
\hline Male & 47,695 & $(52.7)$ & 6,702 & $(100)$ & 2,966 & $(44.3)$ \\
\hline Mean (SD) age (years) & 52.0 & $(10.8)$ & 6,702 & $(100)$ & 52.4 & $(10.4)$ \\
\hline Age (years) & & & 6,702 & $(100)$ & & \\
\hline$<50$ & 36,798 & $(40.7)$ & & & 2,131 & $(31.8)$ \\
\hline $50-<55$ & 15,739 & (17.4) & & & 1,997 & $(29.8)$ \\
\hline $55-<60$ & 16,413 & $(18.1)$ & & & 1,126 & $(16.8)$ \\
\hline $60-<65$ & 10,468 & $(11.6)$ & & & 651 & $(9.7)$ \\
\hline $65-<70$ & 6,561 & $(7.3)$ & & & 428 & $(6.4)$ \\
\hline $70-<75$ & 4,508 & $(5.0)$ & & & 369 & $(5.5)$ \\
\hline \multicolumn{7}{|l|}{ Body measurements } \\
\hline Body mass index & & & 5,924 & $(88.4)$ & & \\
\hline$<-1 \mathrm{SD}$ below the mean & 12,587 & $(13.9)$ & & & 831 & $(14.0)$ \\
\hline-1 to $<-1 / 2$ SD below the mean & 17,793 & (19.7) & & & 1,196 & $(20.2)$ \\
\hline$\geq-1 / 2$ to $\leq 1 / 2$ SD from the mean & 36,066 & $(39.9)$ & & & 2,315 & $(39.1)$ \\
\hline$>1 / 2$ to $1 \mathrm{SD}$ above the mean & 10,744 & (11.9) & & & 678 & $(11.4)$ \\
\hline$>1$ to $2 \mathrm{SD}$ above the mean & 9,803 & $(10.8)$ & & & 649 & $(11.0)$ \\
\hline$>2$ SD above the mean & 3,494 & $(3.9)$ & & & 255 & $(4.3)$ \\
\hline Waist-to-hip ratio & & & 3,918 & $(58.5)$ & & \\
\hline$<-1 \mathrm{SD}$ below the mean & 12,661 & $(14.0)$ & & & 377 & $(9.6)$ \\
\hline-1 to $<-1 / 2 \mathrm{SD}$ below the mean & 17,454 & $(19.3)$ & & & 586 & $(15.0)$ \\
\hline$\geq-1 / 2$ to $\leq 1 / 2$ SD from the mean & 34,932 & $(38.6)$ & & & 1,598 & $(40.8)$ \\
\hline$>1 / 2$ to $1 \mathrm{SD}$ above the mean & 11,901 & $(13.2)$ & & & 585 & $(14.9)$ \\
\hline$>1$ to $2 \mathrm{SD}$ above the mean & 10,535 & $(11.6)$ & & & 587 & $(15.0)$ \\
\hline$>2 \mathrm{SD}$ above the mean & 3,004 & $(3.3)$ & & & 185 & $(4.7)$ \\
\hline A body shape index $\dagger$ & & & 3,854 & $(57.5)$ & & \\
\hline$<-1 \mathrm{SD}$ below the mean & 13,037 & $(14.4)$ & & & 530 & $(13.8)$ \\
\hline-1 to $<-1 / 2 \mathrm{SD}$ below the mean & 15,582 & $(17.2)$ & & & 696 & $(18.1)$ \\
\hline$\geq-1 / 2$ to $\leq 1 / 2$ SD from the mean & 36,221 & $(40.0)$ & & & 1,560 & $(40.5)$ \\
\hline$>1 / 2$ to $1 \mathrm{SD}$ above the mean & 11,947 & $(13.2)$ & & & 546 & $(14.2)$ \\
\hline$>1$ to $2 \mathrm{SD}$ above the mean & 10,810 & (11.9) & & & 397 & $(10.3)$ \\
\hline$>2$ SD above the mean & 2,890 & $(3.2)$ & & & 125 & $(3.2)$ \\
\hline Waist-to-height ratio & & & 3,878 & $(57.9)$ & & \\
\hline$<-1$ SD below the mean & 14,002 & $(15.5)$ & & & 549 & $(14.2)$ \\
\hline-1 to $<-1 / 2 \mathrm{SD}$ below the mean & 16,583 & $(18.3)$ & & & 675 & $(17.4)$ \\
\hline$\geq-1 / 2$ to $\leq 1 / 2$ SD from the mean & 34,394 & $(38.0)$ & & & 1,456 & $(37.5)$ \\
\hline$>1 / 2$ to $1 \mathrm{SD}$ above the mean & 11,571 & $(12.8)$ & & & 524 & $(13.5)$ \\
\hline$>1$ to $2 \mathrm{SD}$ above the mean & 10,722 & $(11.8)$ & & & 494 & $(12.7)$ \\
\hline$>2$ SD above the mean & 3,215 & $(3.6)$ & & & 180 & $(4.6)$ \\
\hline Waist circumference & & & 3,948 & $(58.9)$ & & \\
\hline$<-1 \mathrm{SD}$ below the mean & 13,637 & $(15.1)$ & & & 535 & $(13.6)$ \\
\hline-1 to $<-1 / 2$ SD below the mean & 17,369 & $(19.2)$ & & & 713 & $(18.1)$ \\
\hline$\geq-1 / 2$ to $\leq 1 / 2$ SD from the mean & 34,148 & $(37.7)$ & & & 1,433 & $(36.3)$ \\
\hline$>1 / 2$ to $1 \mathrm{SD}$ above the mean & 11,405 & (12.6) & & & 540 & $(13.7)$ \\
\hline$>1$ to $2 \mathrm{SD}$ above the mean & 10,790 & (11.9) & & & 524 & $(13.3)$ \\
\hline$>2 \mathrm{SD}$ above the mean & 3,138 & $(3.5)$ & & & 203 & $(5.1)$ \\
\hline Hip circumference & & & 3,930 & $(58.6)$ & & \\
\hline$<-1 \mathrm{SD}$ below the mean & 12,359 & $(13.7)$ & & & 640 & $(16.3)$ \\
\hline-1 to $<-1 / 2 \mathrm{SD}$ below the mean & 16,667 & $(18.4)$ & & & 733 & $(18.7)$ \\
\hline
\end{tabular}




\begin{tabular}{l|rrrrrr}
$\geq-1 / 2$ to $\leq 1 / 2$ SD from the mean & 36,717 & $(40.6)$ & & & 1,455 & $(37.0)$ \\
$>>1 / 2$ to 1 SD above the mean & 11,858 & $(13.1)$ & & & 515 & $(13.1)$ \\
$>1$ to 2 SD above the mean & 9,756 & $(10.8)$ & & & 409 & $(10.4)$ \\
$>2 S D$ bbove the mean & 3,130 & $(3.5)$ & & & 178 & $(4.5)$ \\
Oher vascular risk factors & & & & & & \\
Mean (SD) total cholesterol (mmol/l) & 5.81 & $(1.15)$ & 5,690 & $(84.9)$ & 6.06 & $(1.26)$ \\
Median (IQR) total cholesterol (mmol/l) & 5.70 & $(1.50)$ & 5,690 & $(84.9)$ & 5.90 & $(1.60)$ \\
Mean (SD) HDL cholesterol (mmol/l) & 1.43 & $(0.40)$ & 5,591 & $(83.4)$ & 1.52 & $(0.43)$ \\
Median (IQR) HDL cholesterol (mmol/l) & 1.39 & $(0.50)$ & 5,591 & $(83.4)$ & 1.47 & $(0.56)$ \\
Mean (SD) systolic blood pressure (mm Hg) & 136 & $(21)$ & 6,011 & $(89.7)$ & 141 & $(21)$ \\
Taking antihypertensive drugs & 14,367 & $(15.9)$ & 4,202 & $(62.7)$ & 538 & $(12.8)$ \\
Current daily smoker & 22,545 & $(24.9)$ & 6,587 & $(98.3)$ & 1,889 & $(28.7)$ \\
Diabetes & 4,028 & $(4.5)$ & 5,797 & $(86.5)$ & 232 & $(4.0)$
\end{tabular}

Included in the analysis sample, complete data for all analysis measures.

$\$$ Excluded from the analysis sample, missing data for one of more of the analysis variables.

¥ Number of participants with incomplete data who had data for the measure. For ordinal measures, sample size shown next to variable name.

\# Percent of participants with incomplete data who had data for the measure. For ordinal measures percent shown next to variable name

\& Body-mass index (BMI) is the weight in kilograms divided by the square of the height in meters.

$\dagger \mathrm{A}$ body shape index is waist circumference in metres $/\left(\mathrm{BMI}^{2 / 3} *\right.$ height in meters $\left.{ }^{1 / 2}\right)$. 
Table S4. Estimated risk of death from all-causes among females $(\mathrm{N}=42,792)$ according to categorical body measurement categories under partial and full adjustment ${ }^{\mathbf{s}}$

\begin{tabular}{|c|c|c|c|c|c|c|c|c|c|c|}
\hline \multirow{3}{*}{$\begin{array}{l}\text { Body measurement } \\
\text { Body mass index }\left(\mathrm{kg} / \mathrm{m}^{2}\right)^{+}\end{array}$} & \multirow{2}{*}{$\begin{array}{c}\text { Number of } \\
\text { people }\end{array}$} & \multirow{2}{*}{$\begin{array}{c}\text { Number of } \\
\text { deaths }\end{array}$} & \multicolumn{4}{|c|}{ Partially adjusted $^{\#}$} & \multicolumn{4}{|c|}{ Further adjusted $^{\&}$} \\
\hline & & & \multirow[t]{2}{*}{ HR } & \multicolumn{2}{|c|}{$(95 \% \mathrm{CI})$} & \multirow[t]{2}{*}{ p-value } & \multirow[t]{2}{*}{ HR } & \multicolumn{2}{|c|}{$(95 \% \mathrm{CI})$} & \multirow[t]{2}{*}{ p-value } \\
\hline & & & & & & & & & & \\
\hline$<-1$ SD below the mean & 5,935 & 417 & 1.44 & (1.28 & $-1.61)$ & $4 \times 10^{-20}$ & 1.40 & $(1.25$ & $-1.58)$ & $8 \times 10^{-12}$ \\
\hline-1 to $<-1 / 2 \mathrm{SD}$ below the mean & 9,126 & 602 & 1.13 & $(1.02$ & $-1.25)$ & & 1.15 & $(1.04$ & $-1.27)$ & \\
\hline$\geq-1 / 2$ to $\leq 1 / 2$ SD from the mean & 16,325 & 1,219 & 1.00 & & & & 1.00 & & & \\
\hline$>1 / 2$ to $1 \mathrm{SD}$ above the mean & 4,780 & 437 & 1.16 & $(1.04$ & $-1.29)$ & & 1.09 & $(0.98$ & $-1.22)$ & \\
\hline$>1$ to $2 \mathrm{SD}$ above the mean & 4,859 & 470 & 1.43 & $(1.28$ & $-1.59)$ & & 1.29 & $(1.16$ & $-1.44)$ & \\
\hline$>2 \mathrm{SD}$ above the mean & 1,767 & 177 & 1.84 & $(1.57$ & $-2.16)$ & & 1.54 & $(1.31$ & $-1.81)$ & \\
\hline \multicolumn{11}{|l|}{ Waist-to-hip ratio } \\
\hline$<-1 \mathrm{SD}$ below the mean & 6,148 & 338 & 0.79 & $(0.70$ & $-0.89)$ & $4 \times 10^{-26}$ & 0.87 & $(0.77$ & $-0.98)$ & $1 \times 10^{-12}$ \\
\hline-1 to $<-1 / 2 \mathrm{SD}$ below the mean & 8,125 & 599 & 0.88 & $(0.80$ & $-0.97)$ & & 0.93 & $(0.84$ & $-1.02)$ & \\
\hline$\geq-1 / 2$ to $\leq 1 / 2$ SD from the mean & 16,988 & 1,457 & 1.00 & & & & 1.00 & & & \\
\hline$>1 / 2$ to $1 \mathrm{SD}$ above the mean & 5,229 & 488 & 1.30 & $(1.17$ & $-1.44)$ & & 1.23 & $(1.11$ & $-1.37)$ & \\
\hline$>1$ to $2 \mathrm{SD}$ above the mean & 4,618 & 349 & 1.53 & $(1.36$ & $-1.72)$ & & 1.40 & $(1.24$ & $-1.58)$ & \\
\hline$>2 \mathrm{SD}$ above the mean & 1,684 & 91 & 1.82 & $(1.45$ & $-2.27)$ & & 1.60 & $(1.28$ & $-2.00)$ & \\
\hline \multicolumn{11}{|l|}{ A body shape index $\dagger$} \\
\hline$<-1$ SD below the mean & 5,992 & 332 & 0.71 & $(0.63$ & $-0.80)$ & $5 \times 10^{-29}$ & 0.78 & $(0.68$ & $-0.88)$ & $2 \times 10^{-17}$ \\
\hline-1 to $<-1 / 2 \mathrm{SD}$ below the mean & 8,248 & 534 & 0.79 & $(0.71$ & $-0.87)$ & & 0.83 & $(0.75$ & $-0.91)$ & \\
\hline$\geq-1 / 2$ to $\leq 1 / 2$ SD from the mean & 16,983 & 1,495 & 1.00 & & & & 1.00 & & & \\
\hline$>1 / 2$ to $1 \mathrm{SD}$ above the mean & 4,818 & 495 & 1.29 & $(1.16$ & $-1.43)$ & & 1.19 & $(1.08$ & $-1.33)$ & \\
\hline$>1$ to $2 \mathrm{SD}$ above the mean & 4,932 & 372 & 1.39 & $(1.23$ & $-1.56)$ & & 1.32 & $(1.17$ & $-1.48)$ & \\
\hline$>2 \mathrm{SD}$ above the mean & 1,819 & 94 & 1.90 & $(1.52$ & $-2.38)$ & & 1.74 & $(1.39$ & $-2.18)$ & \\
\hline \multicolumn{11}{|l|}{ Waist-to-height ratio } \\
\hline$<-1 \mathrm{SD}$ below the mean & 6,862 & 374 & 1.04 & $(0.92$ & $-1.17)$ & $1 \times 10^{-23}$ & 1.05 & $(0.93$ & $-1.19)$ & $6 \times 10^{-12}$ \\
\hline-1 to $<-1 / 2 \mathrm{SD}$ below the mean & 8,514 & 592 & 0.99 & $(0.90$ & $-1.10)$ & & 1.01 & $(0.91$ & $-1.11)$ & \\
\hline$\geq-1 / 2$ to $\leq 1 / 2$ SD from the mean & 15,219 & 1,258 & 1.00 & & & & 1.00 & & & \\
\hline$>1 / 2$ to $1 \mathrm{SD}$ above the mean & 5,264 & 473 & 1.19 & $(1.07$ & $-1.33)$ & & 1.12 & $(1.01$ & $-1.25)$ & \\
\hline$>1$ to $2 \mathrm{SD}$ above the mean & 5,290 & 494 & 1.56 & $(1.40$ & $-1.74)$ & & 1.43 & $(1.28$ & $-1.59)$ & \\
\hline$>2 \mathrm{SD}$ above the mean & 1,643 & 131 & 2.09 & $(1.73$ & $-2.51)$ & & 1.72 & $(1.43$ & $-2.08)$ & \\
\hline Waist circumference $(\mathrm{cm})$ & & & & & & & & & & \\
\hline$<-1 \mathrm{SD}$ below the mean & 6,715 & 393 & 1.09 & $(0.97$ & $-1.23)$ & $4 \times 10^{-28}$ & 1.10 & $(0.98$ & $-1.24)$ & $7 \times 10^{-15}$ \\
\hline-1 to $<-1 / 2 \mathrm{SD}$ below the mean & 8,499 & 600 & 1.00 & $(0.91$ & $-1.10)$ & & 1.03 & $(0.93$ & $-1.13)$ & \\
\hline$\geq-1 / 2$ to $\leq 1 / 2 \mathrm{SD}$ from the mean & 15,507 & 1,227 & 1.00 & & & & 1.00 & & & \\
\hline$>1 / 2$ to $1 \mathrm{SD}$ above the mean & 5,128 & 459 & 1.20 & $(1.08$ & $-1.34)$ & & 1.14 & $(1.02$ & $-1.27)$ & \\
\hline$>1$ to $2 \mathrm{SD}$ above the mean & 5,307 & 492 & 1.51 & $(1.36$ & $-1.68)$ & & 1.40 & $(1.26$ & $-1.56)$ & \\
\hline$>2 \mathrm{SD}$ above the mean & 1,636 & 151 & 2.32 & $(1.95$ & $-2.75)$ & & 1.92 & $(1.61$ & $-2.29)$ & \\
\hline Hip circumference $(\mathbf{c m})$ & & & & & & & & & & \\
\hline$<-1 \mathrm{SD}$ below the mean & 5,716 & 414 & 1.52 & $(1.35$ & $-1.71)$ & $4 \times 10^{-20}$ & 1.45 & $(1.29$ & $-1.63)$ & $7 \times 10^{-13}$ \\
\hline-1 to $<-1 / 2$ SD below the mean & 8,358 & 549 & 1.14 & $(1.03$ & $-1.26)$ & & 1.15 & $(1.04$ & $-1.28)$ & \\
\hline$\geq-1 / 2$ to $\leq 1 / 2$ SD from the mean & 17,334 & 1,251 & 1.00 & & & & 1.00 & & & \\
\hline$>1 / 2$ to $1 \mathrm{SD}$ above the mean & 5,065 & 433 & 1.07 & $(0.96$ & $-1.20)$ & & 1.03 & $(0.92$ & $-1.15)$ & \\
\hline$>1$ to $2 \mathrm{SD}$ above the mean & 4,586 & 491 & 1.39 & $(1.25$ & $-1.54)$ & & 1.29 & $(1.16$ & $-1.43)$ & \\
\hline$>2$ SD above the mean & 1,733 & 184 & 1.71 & $(1.46$ & $-2.00)$ & & 1.47 & $(1.25$ & $-1.72)$ & \\
\hline Waist \& hip circumference & & & & & & & & & & \\
\hline Waist & & & & & & & & & & \\
\hline$<-1 \mathrm{SD}$ below the mean & 6,715 & 393 & 0.72 & $(0.62$ & $-0.83)$ & $2 \times 10^{-24}$ & 0.79 & $(0.68$ & $-0.91)$ & $2 \times 10^{-13}$ \\
\hline-1 to $<-1 / 2 \mathrm{SD}$ below the mean & 8,499 & 600 & 0.83 & $(0.74$ & $-0.92)$ & & 0.88 & $(0.79$ & $-0.98)$ & \\
\hline$\geq-1 / 2$ to $\leq 1 / 2$ SD from the mean & 15,507 & 1,227 & 1.00 & & & & 1.00 & & & \\
\hline$>1 / 2$ to $1 \mathrm{SD}$ above the mean & 5,128 & 459 & 1.30 & $(1.15$ & $-1.46)$ & & 1.21 & $(1.08$ & $-1.37)$ & \\
\hline$>1$ to $2 \mathrm{SD}$ above the mean & 5,307 & 492 & 1.67 & $(1.46$ & $-1.91)$ & & 1.52 & $(1.32$ & $-1.74)$ & \\
\hline$>2 \mathrm{SD}$ above the mean & 1,636 & 151 & 2.67 & $(2.14$ & $-3.34)$ & & 2.16 & $(1.72$ & $-2.71)$ & \\
\hline Hip & & & & & & & & & & \\
\hline$<-1 \mathrm{SD}$ below the mean & 5,716 & 414 & 1.95 & $(1.69$ & $-2.25)$ & $1 \times 10^{-18}$ & 1.72 & $(1.49$ & $-1.98)$ & $5 \times 10^{-12}$ \\
\hline-1 to $<-1 / 2 \mathrm{SD}$ below the mean & 8,358 & 549 & 1.33 & $(1.19$ & $-1.48)$ & & 1.27 & $(1.14$ & $-1.42)$ & \\
\hline$\geq-1 / 2$ to $\leq 1 / 2$ SD from the mean & 17,334 & 1,251 & 1.00 & & & & 1.00 & & & \\
\hline$>1 / 2$ to $1 \mathrm{SD}$ above the mean & 5,065 & 433 & 0.90 & $(0.80$ & $-1.01)$ & & 0.91 & $(0.81$ & $-1.02)$ & \\
\hline$>1$ to $2 \mathrm{SD}$ above the mean & 4,586 & 491 & 0.96 & $(0.84$ & $-1.09)$ & & 0.97 & $(0.85$ & $-1.11)$ & \\
\hline$>2 \mathrm{SD}$ above the mean & 1,733 & 184 & 0.87 & $(0.71$ & $-1.07)$ & & 0.89 & $(0.72$ & $-1.09)$ & \\
\hline
\end{tabular}

\$ HR hazard ratio. CI confidence interval. Estimated separately for males and females using Cox proportional hazards models, stratified by cohort, with age at death/censoring as the time variable.

\# Adjusted for age at baseline.

\& Adjusted for age at baseline, log of total cholesterol (mmol/1), log of HDL cholesterol (mmol/l), systolic blood pressure $(\mathrm{mm} \mathrm{Hg})$, taking antihypertensive drugs, current daily smoker (cigarettes, cigars/cigarillos or pipe), diabetes, and an interaction between age at baseline and log of HDL cholesterol (mmol/l).

Body-mass index (BMI) is the weight in kilograms divided by the square of the height in meters.

$\dagger \mathrm{A}$ body shape index is waist circumference in metres/(BMI ${ }^{2 / 3} *$ height in meters $\left.{ }^{1 / 2}\right)$. 
Table S5. Estimated risk of death from all-causes among males $(\mathrm{N}=47,695)$ according to categorical body measurement categories under partial and full adjustment $\mathbf{t}^{\$}$

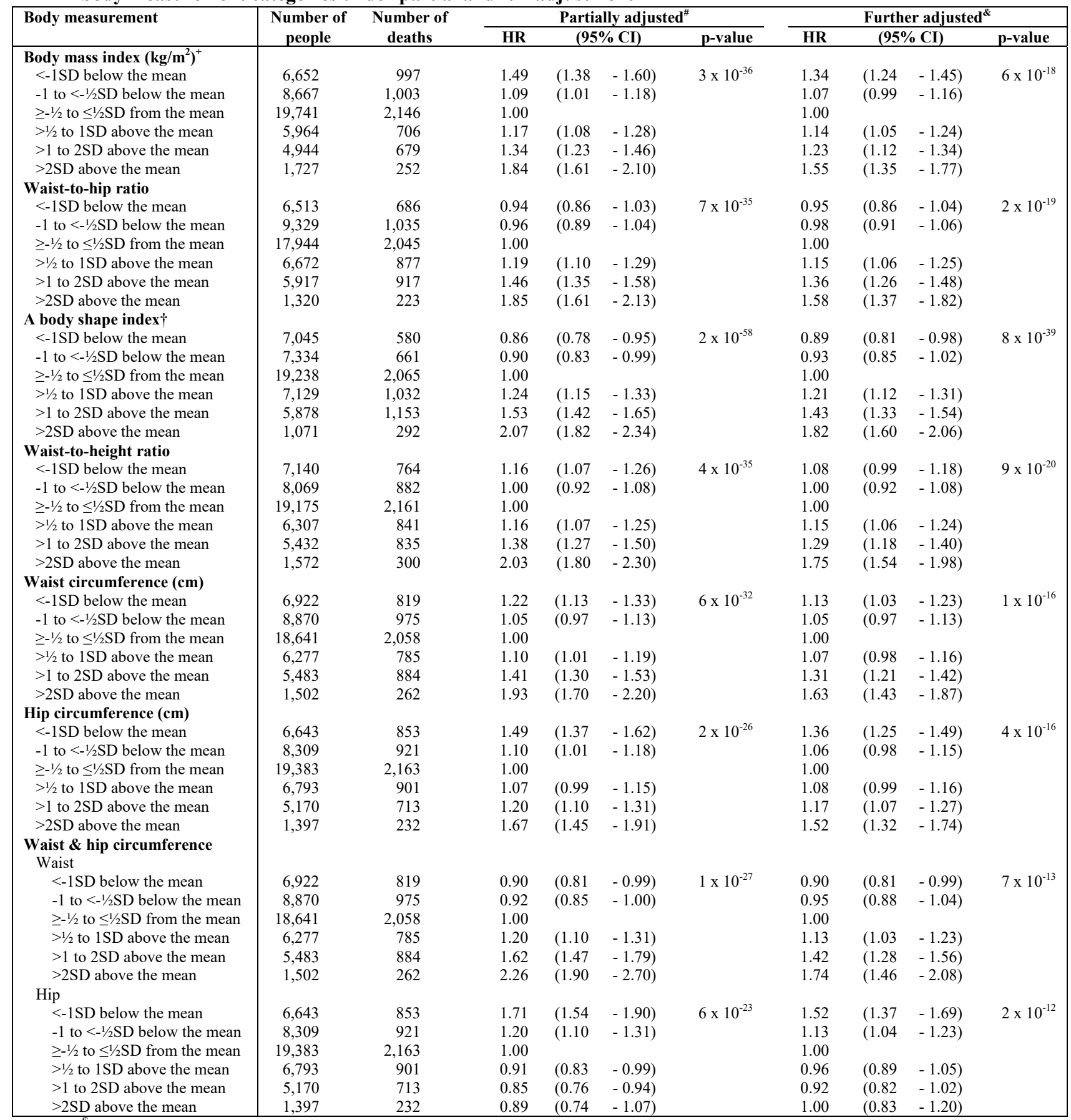

\$ HR hazard ratio. CI confidence interval. Estimated separately for males and females using Cox proportional hazards models, stratified by cohort, with age at death/censoring as the time variable. ${ }^{\#}$ Adjusted for age at baseline. \& Adjusted for age at baseline, log of total cholesterol ( $\mathrm{mmol} / \mathrm{l}), \log$ of HDL cholesterol (mmol/1), systolic blood pressure $(\mathrm{mm} \mathrm{Hg})$, taking antihypertensive drugs, current daily smoker (cigarettes, cigars/cigarillos or pipe), diabetes, and an interaction between age at baseline and current daily smoker. Body-mass index (BMI) is the weight in kilograms divided by the square of the height in meters. $\dagger$ A body shape index is waist circumference in metres/ $\left(\mathrm{BMI}^{2 / 3} *\right.$ height in meters $\left.{ }^{1 / 2}\right)$. 
Table S6. Estimated risk of death from cardiovascular disease among females $(\mathrm{N}=42,792)$ according to categorical body measurement categories under partial and full adjustment ${ }^{\mathrm{s}}$

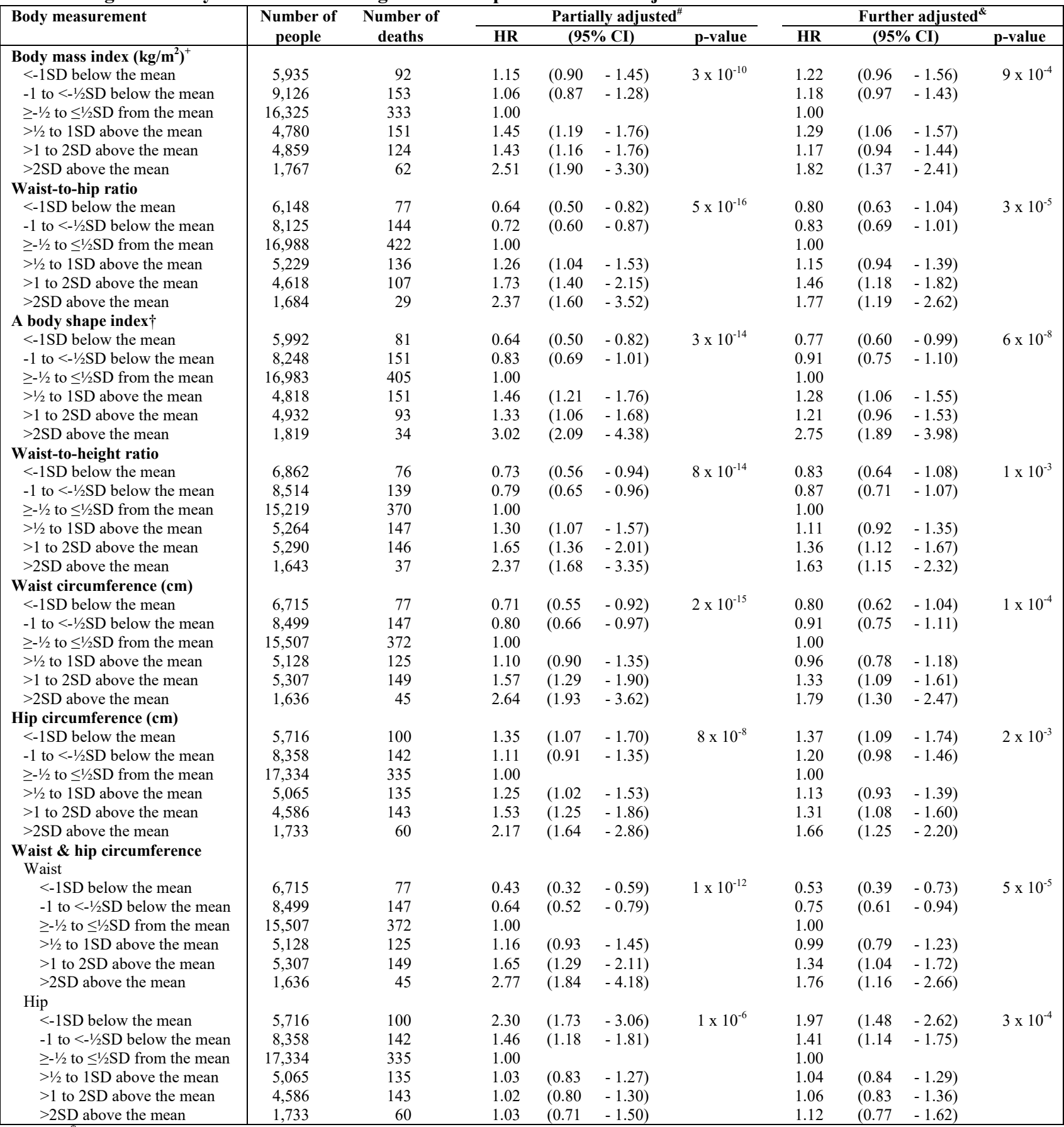

$\$$ HR hazard ratio. CI confidence interval. Estimated separately for males and females using Cox proportional hazards models, stratified by cohort, with age at death/censoring as the time variable. ${ }^{\#}$ Adjusted for age at baseline. \& Adjusted for age at baseline, log of total cholesterol ( $\mathrm{mmol} / \mathrm{l}), \log$ of HDL cholesterol (mmol/l), systolic blood pressure $(\mathrm{mm} \mathrm{Hg})$, taking antihypertensive drugs, current daily smoker (cigarettes, cigars/cigarillos or pipe), diabetes, and an interaction between age at baseline and systolic blood pressure. Body-mass index (BMI) is the weight in kilograms divided by the square of the height in meters. $\dagger \mathrm{A}$ body shape index is waist circumference in metres $/\left(\mathrm{BMI}^{2 / 3} *\right.$ height in meters $\left.{ }^{1 / 2}\right)$. 
Table S7. Estimated risk of death from cardiovascular disease among males $(N=47,695)$ according to categorical body measurement categories under partial and full adjustment ${ }^{\mathrm{s}}$

\begin{tabular}{|c|c|c|c|c|c|c|c|c|c|c|}
\hline \multirow[t]{2}{*}{ Body measurement } & \multirow{2}{*}{$\begin{array}{c}\text { Number of } \\
\text { people }\end{array}$} & \multirow{2}{*}{$\begin{array}{c}\text { Number of } \\
\text { deaths }\end{array}$} & \multicolumn{4}{|c|}{ Partially adjusted $^{\#}$} & \multicolumn{4}{|c|}{ Further adjusted $^{\&}$} \\
\hline & & & \multirow[t]{2}{*}{ HR } & \multicolumn{2}{|c|}{$(95 \% \mathrm{CI})$} & \multirow[t]{2}{*}{ p-value } & \multirow[t]{2}{*}{ HR } & \multicolumn{2}{|c|}{$(95 \% \mathrm{CI})$} & \multirow[t]{2}{*}{ p-value } \\
\hline Body mass index $\left(\mathrm{kg} / \mathrm{m}^{2}\right)^{+}$ & & & & & & & & & & \\
\hline$<-1$ SD below the mean & 6,652 & 212 & 1.07 & (0.91 & $-1.25)$ & $8 \times 10^{-12}$ & 1.17 & (0.99 & $-1.37)$ & $5 \times 10^{-3}$ \\
\hline-1 to $<-1 / 2$ SD below the mean & 8,667 & 272 & 0.99 & $(0.86$ & $-1.14)$ & & 1.07 & $(0.93$ & $-1.24)$ & \\
\hline$\geq-1 / 2$ to $\leq 1 / 2$ SD from the mean & 19,741 & 651 & 1.00 & & & & 1.00 & & & \\
\hline$>1 / 2$ to $1 \mathrm{SD}$ above the mean & 5,964 & 220 & 1.22 & $(1.04$ & $-1.42)$ & & 1.12 & $(0.96$ & $-1.30)$ & \\
\hline$>1$ to $2 \mathrm{SD}$ above the mean & 4,944 & 222 & 1.44 & $(1.23$ & $-1.68)$ & & 1.19 & $(1.02$ & $-1.39)$ & \\
\hline$>2 \mathrm{SD}$ above the mean & 1,727 & 85 & 2.12 & $(1.69$ & $-2.67)$ & & 1.53 & $(1.21$ & $-1.93)$ & \\
\hline \multicolumn{11}{|l|}{ Waist-to-hip ratio } \\
\hline$<-1 \mathrm{SD}$ below the mean & 6,513 & 170 & 0.78 & $(0.65$ & $-0.93)$ & $2 \times 10^{-26}$ & 0.93 & $(0.78$ & $-1.11)$ & $3 \times 10^{-9}$ \\
\hline-1 to $<-1 / 2 \mathrm{SD}$ below the mean & 9,329 & 235 & 0.73 & $(0.63$ & $-0.85)$ & & 0.81 & $(0.69$ & $-0.94)$ & \\
\hline$\geq-1 / 2$ to $\leq 1 / 2$ SD from the mean & 17,944 & 607 & 1.00 & & & & 1.00 & & & \\
\hline$>1 / 2$ to $1 \mathrm{SD}$ above the mean & 6,672 & 280 & 1.30 & $(1.13$ & $-1.50)$ & & 1.21 & $(1.04$ & $-1.39)$ & \\
\hline$>1$ to $2 \mathrm{SD}$ above the mean & 5,917 & 300 & 1.64 & $(1.42$ & $-1.89)$ & & 1.40 & $(1.21$ & $-1.62)$ & \\
\hline$>2 \mathrm{SD}$ above the mean & 1,320 & 70 & 2.04 & $(1.59$ & $-2.63)$ & & 1.50 & $(1.16$ & $-1.93)$ & \\
\hline \multicolumn{11}{|l|}{ A body shape index $\dagger$} \\
\hline$<-1 \mathrm{SD}$ below the mean & 7,045 & 160 & 0.88 & $(0.74$ & $-1.06)$ & $1 \times 10^{-24}$ & 1.01 & $(0.84$ & $-1.21)$ & $2 \times 10^{-15}$ \\
\hline$\geq-1 / 2$ to $\leq 1 / 2 \mathrm{SD}$ from the mean & 19,238 & 600 & 1.00 & & & & 1.00 & & & \\
\hline$>1 / 2$ to $1 \mathrm{SD}$ above the mean & 7,129 & 293 & 1.20 & $(1.04$ & $-1.38)$ & & 1.16 & $(1.01$ & $-1.34)$ & \\
\hline$>1$ to $2 \mathrm{SD}$ above the mean & 5,878 & 367 & 1.65 & $(1.44$ & $-1.88)$ & & 1.51 & $(1.32$ & $-1.73)$ & \\
\hline$>2 \mathrm{SD}$ above the mean & 1,071 & 91 & 2.21 & $(1.77$ & $-2.77)$ & & 1.94 & $(1.55$ & $-2.44)$ & \\
\hline \multicolumn{11}{|l|}{ Waist-to-height ratio } \\
\hline$<-1 \mathrm{SD}$ below the mean & 7,140 & 167 & 0.96 & $(0.80$ & $-1.14)$ & $6 \times 10^{-30}$ & 1.08 & $(0.90$ & $-1.30)$ & $4 \times 10^{-11}$ \\
\hline-1 to $<-1 / 2 \mathrm{SD}$ below the mean & 8,069 & 208 & 0.84 & $(0.72$ & $-0.99)$ & & 0.93 & $(0.79$ & $-1.09)$ & \\
\hline$\geq-1 / 2$ to $\leq 1 / 2 \mathrm{SD}$ from the mean & 19,175 & 606 & 1.00 & & & & 1.00 & & & \\
\hline$>1 / 2$ to $1 \mathrm{SD}$ above the mean & 6,307 & 294 & 1.44 & $(1.25$ & $-1.66)$ & & 1.31 & $(1.14$ & $-1.51)$ & \\
\hline$>1$ to $2 \mathrm{SD}$ above the mean & 5,432 & 281 & 1.66 & $(1.44$ & $-1.92)$ & & 1.40 & $(1.21$ & $-1.62)$ & \\
\hline$>2 \mathrm{SD}$ above the mean & 1,572 & 106 & 2.66 & $(2.16$ & $-3.29)$ & & 1.95 & $(1.58$ & $-2.42)$ & \\
\hline Waist circumference (cm) & & & & & & & & & & \\
\hline$<-1 \mathrm{SD}$ below the mean & 6,922 & 187 & 1.00 & $(0.85$ & $-1.18)$ & $9 \times 10^{-21}$ & 1.11 & $(0.94$ & $-1.32)$ & $9 \times 10^{-7}$ \\
\hline-1 to $<-1 / 2 \mathrm{SD}$ below the mean & 8,870 & 228 & 0.86 & $(0.74$ & $-1.00)$ & & 0.94 & $(0.80$ & $-1.09)$ & \\
\hline$\geq-1 / 2$ to $\leq 1 / 2$ SD from the mean & 18,641 & 600 & 1.00 & & & & 1.00 & & & \\
\hline$>1 / 2$ to $1 \mathrm{SD}$ above the mean & 6,277 & 260 & 1.23 & $(1.07$ & $-1.43)$ & & 1.13 & $(0.97$ & $-1.31)$ & \\
\hline$>1$ to $2 \mathrm{SD}$ above the mean & 5,483 & 298 & 1.60 & $(1.39$ & $-1.84)$ & & 1.35 & $(1.17$ & $-1.55)$ & \\
\hline-1 to $<-1 / 2$ SD below the mean & 8,309 & 244 & 1.03 & $(0.88$ & $-1.19)$ & & 1.07 & $(0.92$ & $-1.24)$ & \\
\hline$\geq-1 / 2$ to $\leq 1 / 2$ SD from the mean & 19,383 & 627 & 1.00 & & & & 1.00 & & & \\
\hline$>1 / 2$ to $1 \mathrm{SD}$ above the mean & 6,793 & 282 & 1.13 & $(0.98$ & $-1.30)$ & & 1.09 & $(0.94$ & $-1.25)$ & \\
\hline$>1$ to $2 \mathrm{SD}$ above the mean & 5,170 & 235 & 1.36 & $(1.17$ & $-1.58)$ & & 1.21 & $(1.04$ & $-1.41)$ & \\
\hline$>2 \mathrm{SD}$ above the mean & 1,397 & 82 & 2.04 & $(1.62$ & $-2.58)$ & & 1.68 & $(1.33$ & $-2.12)$ & \\
\hline Waist \& hip circumference & & & & & & & & & & \\
\hline Waist & & & & & & & & & & \\
\hline$<-1 \mathrm{SD}$ below the mean & 6,922 & 187 & 0.73 & $(0.59$ & $-0.89)$ & $2 \times 10^{-16}$ & 0.88 & $(0.72$ & $-1.09)$ & $7 \times 10^{-5}$ \\
\hline-1 to $<-1 / 2 \mathrm{SD}$ below the mean & 8,870 & 228 & 0.75 & $(0.63$ & $-0.88)$ & & 0.85 & $(0.72$ & $-1.00)$ & \\
\hline$\geq-1 / 2$ to $\leq 1 / 2 \mathrm{SD}$ from the mean & 18,641 & 600 & 1.00 & & & & 1.00 & & & \\
\hline$>1 / 2$ to $1 \mathrm{SD}$ above the mean & 6,277 & 260 & 1.36 & $(1.16$ & $-1.59)$ & & 1.20 & $(1.02$ & $-1.40)$ & \\
\hline$>1$ to $2 \mathrm{SD}$ above the mean & 5,483 & 298 & 1.85 & $(1.55$ & $-2.20)$ & & 1.46 & $(1.22$ & $-1.73)$ & \\
\hline$>2 \mathrm{SD}$ above the mean & 1,502 & 89 & 2.55 & $(1.87$ & $-3.47)$ & & 1.70 & $(1.25$ & $-2.32)$ & \\
\hline Hip & & & & & & & & & & \\
\hline$<-1 \mathrm{SD}$ below the mean & 6,643 & 192 & 1.72 & $(1.39$ & $-2.12)$ & $6 \times 10^{-6}$ & 1.51 & $(1.22$ & $-1.87)$ & $3 \times 10^{-3}$ \\
\hline-1 to $<-1 / 2$ SD below the mean & 8,309 & 244 & 1.26 & $(1.07$ & $-1.48)$ & & 1.19 & $(1.01$ & $-1.39)$ & \\
\hline$\geq-1 / 2$ to $\leq 1 / 2$ SD from the mean & 19,383 & 627 & 1.00 & & & & 1.00 & & & \\
\hline$>1 / 2$ to $1 \mathrm{SD}$ above the mean & 6,793 & 282 & 0.88 & $(0.75$ & $-1.02)$ & & 0.94 & $(0.81$ & $-1.10)$ & \\
\hline$>1$ to $2 \mathrm{SD}$ above the mean & 5,170 & 235 & 0.84 & $(0.70$ & $-1.02)$ & & 0.92 & $(0.76$ & $-1.11)$ & \\
\hline$>2 \mathrm{SD}$ above the mean & 1,397 & 82 & 0.96 & $(0.70$ & $-1.32)$ & & 1.11 & $(0.81$ & $-1.52)$ & \\
\hline
\end{tabular}

\$ HR hazard ratio. CI confidence interval. Estimated separately for males and females using Cox proportional hazards models, stratified by cohort, with age at death/censoring as the time variable. ${ }^{*}$ Adjusted for age at baseline. Adjusted for age at baseline, log of total cholesterol ( $\mathrm{mmol} / \mathrm{l}), \log$ of HDL cholesterol (mmol/l), systolic blood pressure ( $\mathrm{mm} \mathrm{Hg}$ ), taking antihypertensive drugs, current daily smoker (cigarettes, cigars/cigarillos or pipe), diabetes, and an interaction between age at baseline and current daily smoker. Body-mass index (BMI) is the weight in kilograms divided by the square of the height in meters. $\dagger \mathrm{A}$ body shape index is waist circumference in metres $/\left(\mathrm{BMI}^{2 / 3} *\right.$ height in meters $\left.{ }^{1 / 2}\right)$. 
Figure S1. Risk of all-cause death according to obesity measures by sex and daily smoking status for all participants, estimated after adjustment for cardiovascular risk factors.

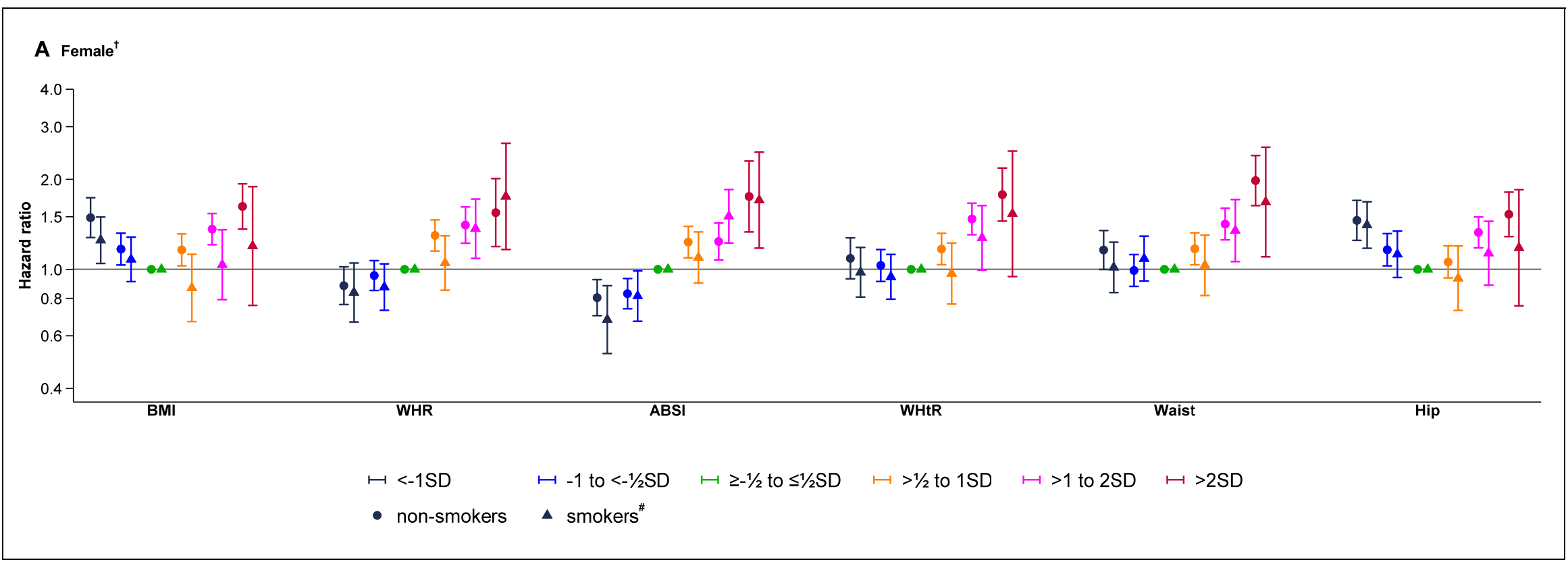




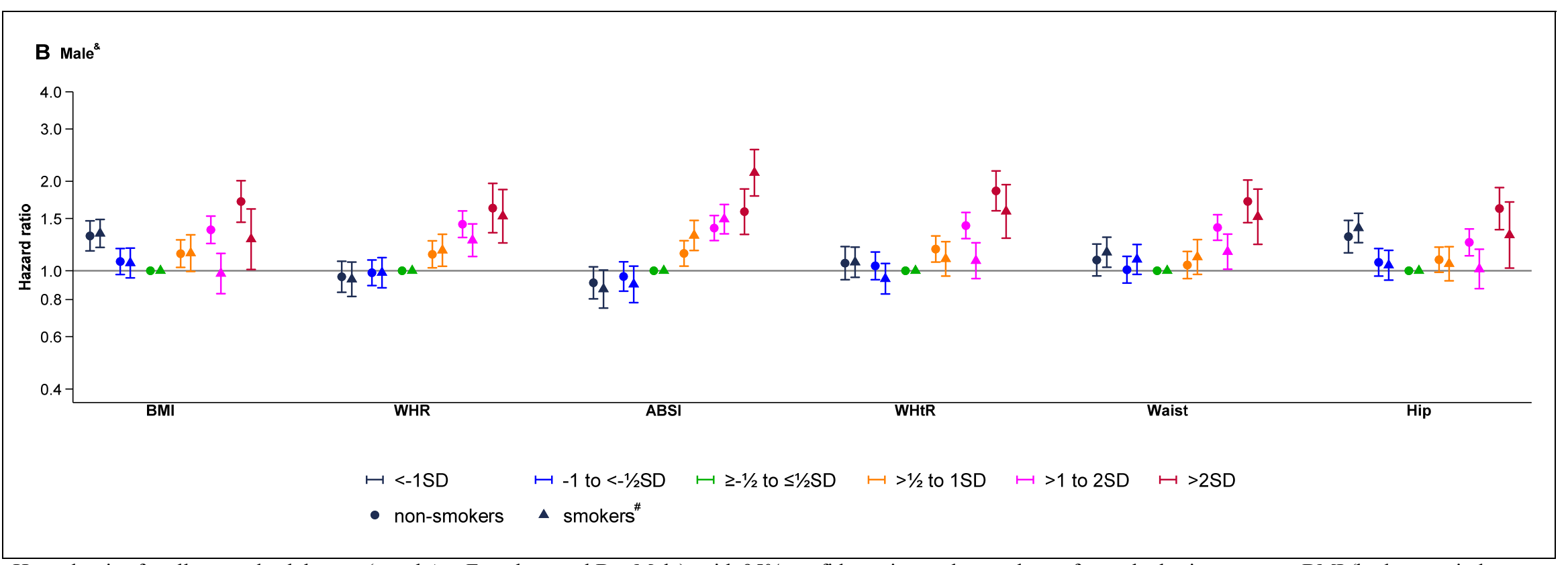

Hazard ratios for all-cause death by sex (panel A = Female; panel B = Male), with 95\% confidence intervals, are shown for each obesity measure: BMI (body mass index the weight in kilograms divided by the square of the height in meters), WHR (weight-to-hip ratio), ABSI (a body shape index: waist circumference in metres/(BMI ${ }^{2 / 3}$

*height in meters ${ }^{1 / 2}$ )), WHtR (weight-to-height ratio), WC and HC). Vertical lines indicate $95 \%$ confidence intervals. Reference category for each obesity measure was $-1 / 2$ standard deviation (SD) to $1 / 2 \mathrm{SD}$ from the sample sex-specific mean calculated using all participants. Risks were estimated from Cox proportional hazards models, stratified by cohort, and adjusted for age at baseline, log of total cholesterol (mmol/l), log of high density lipoprotein (HDL) cholesterol (mmol/l), systolic blood pressure (mm Hg), taking antihypertensive drugs, diabetes, current daily smoker and interaction between obesity measure and current daily smoker, and all interactions between age and other baseline measures that were statistically significant $(\mathrm{p}<0.001)$. $\uparrow$ Additionally adjusted for an interaction between age at baseline and log of HDL cholesterol $(\mathrm{mmol} / \mathrm{l})$. Additionally adjusted for an interaction between age at baseline and current daily smoker. ${ }^{\#}$ Estimates do not include main effect of smoking to make the risk comparable to the non-smokers. 